\title{
Fully General Relativistic Simulations of Black Hole-Neutron Star Mergers
}

\author{
Zachariah B. Etienne, ${ }^{1, \text { * }}$ Joshua A. Faber,, , 团 Yuk Tung Liu, ${ }^{1}$ \\ Stuart L. Shapiro, ${ }^{1, \text { f }}$ Keisuke Taniguchi, ${ }^{1}$ and Thomas W. Baumgarte ${ }^{2, \text { S }}$ \\ ${ }^{1}$ Department of Physics, University of Illinois at Urbana-Champaign, Urbana, IL 61801 \\ ${ }^{2}$ Department of Physics and Astronomy, Bowdoin College, Brunswick, ME 04011
}

\begin{abstract}
Black hole-neutron star (BHNS) binaries are expected to be among the leading sources of gravitational waves observable by ground-based detectors, and may be the progenitors of short-hard gamma ray bursts (SGRBs) as well. We discuss our new fully general relativistic calculations of merging BHNS binaries, which use high-accuracy, low-eccentricity, conformal thin-sandwich configurations as initial data. Our evolutions are performed using the moving puncture method and include a fully relativistic, high-resolution shock-capturing hydrodynamics treatment. Focusing on systems in which the neutron star is irrotational and the black hole is nonspinning with a 3:1 mass ratio, we investigate the inspiral, merger, and disk formation in the system. We find that the vast majority of material is promptly accreted and no more than $3 \%$ of the neutron star's rest mass is ejected into a tenuous, gravitationally bound disk. We find similar results for mass ratios of 2:1 and 1:1, even when we reduce the NS compaction in the 2:1 mass ratio case. These ambient disks reach temperatures suitable for triggering SGRBs, but their masses may be too small to produce the required total energy output. We measure gravitational waveforms and compute the effective strain in frequency space, finding measurable differences between our waveforms and those produced by binary black hole mergers within the advanced LIGO band. These differences appear at frequencies corresponding to the emission that occurs when the NS is tidally disrupted and accreted by the black hole. The resulting information about the radius of the neutron star may be used to constrain the neutron star equation of state.
\end{abstract}

PACS numbers: 04.25.D-,04.25.dk,04.30.-w

\section{INTRODUCTION}

Mergers of compact binaries, consisting either of neutron stars (NS) or black holes (BH), are expected to be among the most promising sources of gravitational waves detectable by ground-based laser interferometers like LIGO [1, 2], VIRGO [3, 4], GEO [5], and TAMA [6, 7], as well as by the proposed space-based interferometers LISA [8] and DECIGO [9]. Theoretical models indicate that a neutron star-neutron star (NSNS) 10, 11, 12, 13, 14, 15, 16 or black hole-neutron star (BHNS) 16, 17, 18, 19, 20, 21 merger may result in a hot, massive disk around a $\mathrm{BH}$, whose temperatures and densities could be sufficient to trigger a short-hard gamma-ray burst (SGRB). Indeed, SGRBs have been repeatedly associated with galaxies with extremely low star formation rates (see 22] and references therein for a review), indicating that the source is likely to involve an evolved population, rather than main sequence stars.

Modeling the inspiral, coalescence and merger of compact binaries requires fully general relativistic dynamical

\footnotetext{
*Electronic address: zetienne@uiuc.edu

${ }^{\dagger}$ National Science Foundation (NSF) Astronomy and Astrophysics Postdoctoral Fellow.; Current Address: School of Mathematical Sciences, Rochester Institute of Technology, Rochester, NY 14623 $¥$ Also at Department of Astronomy and NCSA, University of Illinois at Urbana-Champaign, Urbana, IL 61801

$\S$ Also at Department of Physics, University of Illinois at UrbanaChampaign, Urbana, IL 61801
}

simulations, and has been a long-standing goal of numerical relativity (see [23] for a review). Historically, the first successful dynamical simulations of compact binaries involved NSNS binaries [13, 14, 15, 24, 25, 26, 27, 28]). A breakthrough in the simulations of BHBH binaries occurred more recently [29, 30, 31]. Simulations of BHNS binaries, on the other hand, have so far lagged behind - perhaps because they combine the difficulties associated with black hole singularities with the subtleties of relativistic hydrodynamics. To date the only fully selfconsistent dynamical simulations of BHNS inspiral and coalescence are those of Shibata and Uryū [19, 20] (hereafter SU) and Shibata and Taniguchi [21] (hereafter ST).

Over the past years, we have systematically developed the tools necessary to simulate the inspiral and merger of BHNS binaries, including the tidal disruption of the neutron stars and the potential formation of an accretion disk. As reviewed below, we have constructed quasiequilibrium initial data describing relativistic BHNS binaries in quasicircular orbits [32, 33, 34, 35, 36], and performed preliminary relativistic dynamical simulations by assuming several simplifying approximations [17, 18]. We also demonstrated and tested how the numerical techniques adopted in many recent $\mathrm{BHBH}$ puncture simulations can be combined with relativistic hydrodynamics to evolve quasiequilibrium initial data that are provided only in the black hole exterior 37, 38]. In this paper we report on our first fully self-consistent, dynamical simulations of BHNS binaries.

For the initial data, we have adopted a hierarchical approach to construct quasiequilibrium models of BHNS 
in quasicircular orbit. We began by making a number of simplifying assumptions, and have relaxed these assumptions step by step ([32, 33, 34, 35, 36], cf. [39, 40] for other BHNS initial data). Our current models, which we adopt as initial data for the dynamical simulations described in this paper, are solutions to Einstein's constraint equations in the conformal thin-sandwich (CTS) decomposition. We model the neutron star as an irrotational $\Gamma=2$ polytrope, and impose the black hole equilibrium boundary conditions of Cook and Pfeiffer [41] on the black hole horizon, to approximate an irrotational BH. In our most recent paper [36], we adopted the methods of Caudill et.al. [42] to construct irrotational black holes more accurately, and found closer agreement with post-Newtonian results. These improved initial data will be incorporated into our next set of dynamical calculations.

For the purpose of comparison we point out that the dynamical simulations of SU and ST adopt initial data that are different from ours. Both approaches lead to valid solutions to Einstein's constraint equations, but the solutions may be physically distinct. Specifically, our initial data use the CTS decomposition, which allows us to impose an approximate helical Killing vector on the spacetime and thereby set to zero several time derivatives of the field variables in a corotating frame. For example, we impose all conditions $\partial_{t} \tilde{\gamma}_{i j}=0$, where $\tilde{\gamma}_{i j}$ is the conformally related spatial metric, and these immediately yield a relation between the components of the extrinsic curvature and the shift vector [see Eq. (27)]. By contrast, SU and ST do not impose these conditions, but instead use the conformal transverse-traceless (CTT) decomposition to obtain the extrinsic curvature on the initial slice. However, they do employ the assumption of a helical Killing vector to construct a lapse and shift, (cf., [43] who use a similar approach for BHBHs) and these gauge quantities are used to solve the quasiequilibrium fluid equations for the neutron star. They are also used to compute the matter source terms appearing in the constraint equations. For example, SU and ST take the divergence of Eq. (27) to generate three equations for the shift. In addition, they model the BH as a "puncture" (see [44, 45, 46]), whereas we excise the $\mathrm{BH}$ interior and impose the equilibrium boundary conditions on the apparent horizon to force the $\mathrm{BH}$ to be stationary, at least momentarily. Therefore, one might speculate that our CTS initial data may represent quasiequilibrium BHNSs in quasicircular orbit more faithfully than SU and ST's initial data. In this paper, we present evidence that the details of the initial data have a noticeable impact on the outcome of the merger - including the disk mass - which may explain some of the differences between the findings of SU and ST and ours.

In most dynamical simulations of BHNS binaries to date, the self-gravity of the NS and/or the tidal gravity of the $\mathrm{BH}$ are treated in a Newtonian or post-Newtonian framework (see, e.g., 47, 48, 49, 50, 51]; see also 52] who performed fully relativistic simulations of head-on collisions). In many calculations, especially those with an initial mass ratio $q=M_{\mathrm{BH}} / M_{\mathrm{NS}} \lesssim 3$, significant disks are formed after the NS is disrupted, and for very stiff nuclear equations of state (EOSs), the core of the NS may survive the initial mass transfer episode and remain bound. These findings contrast with some semi-analytic relativistic arguments that suggest that it is very difficult to form disks with appreciable masses in the merger of BHNS binaries [53.

Using our earlier initial data 32, 33], which assumed extreme mass ratios with $q \gg 1$, we performed simulations of BHNS merger in an approximate relativistic framework [17, 18]. In particular, we assumed that the spatial metric remains conformally flat throughout the evolution (see [54, 55]). Though this approach only allows for crude estimates, we found that mergers of irrotational BHNS binaries may lead to disks of masses up to $0.3 M_{\odot}$, with sufficient heating to emit the neutrino fluxes that are required to launch a gamma-ray burst. In their fully relativistic BHNS simulations, SU later found disk masses in the range of $0.1-0.3 M_{\odot}$ for corotating NSs, and ST found smaller disk masses of $0.04-0.16 M_{\odot}$ for more realistic irrotational NSs.

In preparation for our fully relativistic dynamical simulations of BHNS merger, we demonstrated in 37, 38 that the moving puncture method (see [30, 31] as well as numerous later publications), which has proven extremely useful for BHBH simulations, can be adopted for BHNS simulations and conformal thin-sandwich initial data. Two conceptional issues were addressed, namely the inclusion of relativistic hydrodynamics into these simulations, and the fact that moving puncture simulations require initial data everywhere, while our conformal thinsandwich initial data excise the black hole interior and hence provide data only in the black hole exterior.

In contrast to the original dynamical puncture simulations [56, 57], in which the puncture was forced to remain at a fixed coordinate location, the "moving puncture" approach allows the punctures to move freely through the computational grid. This method is typically used in the context of the BSSN formulation [58, 59], coupled to a "Gamma-driving" shift [60] and a "1+log" slicing condition 61]. Geometrical arguments show that, with this slicing condition, dynamical simulations approach limit surfaces of finite areal radius around black hole singularities, but never reach the singularity itself (see 62, 63, 64, 65]). These findings provide insight into why moving puncture simulations can possibly be successful, and also suggest that it may be possible to incorporate relativistic hydrodynamics into these simulations. Since the simulations only cover regular regions of the spacetime, the hydrodynamic flow never encounters any black hole singularities. In [37] we demonstrated that with only very minor modifications, our high-resolution shockcapturing (HRSC) relativistic hydrodynamics algorithm (see 66]) can indeed be used together with the moving puncture method to model accretion onto black holes.

Part of the appeal of the moving puncture approach 
stems from the fact that it does not require an excision of the black hole interior. Accordingly, this method requires initial data everywhere, both in the BH's exterior and interior. Most dynamical moving puncture simulations to date have therefore adopted the puncture method also in the construction of the initial data (see e.g., [46, 67]). As discussed above, CTS initial data are generally believed to be very good approximations of true quasiequilibrium states, but solving the CTS equations usually involves excising the $\mathrm{BH}$ interior, so that the resulting data exist only in the $\mathrm{BH}$ exterior. By definition, no physical information can propagate from the $\mathrm{BH}$ interior to the exterior, and we have recently demonstrated that even unphysical, constraint-violating noise ("junk") does not leave the $\mathrm{BH}(37,38$, see also 68] ) in numerical evolutions employing the BSSN formulation and moving puncture gauge conditions (modulo certain smoothness restrictions on the junk data near the horizon). Thus, the $\mathrm{BH}$ interiors in CTS initial data can be filled with "junk" without affecting the external spacetime, enabling us to evolve our quasiequilibrium BHNS initial data via the moving puncture formalism.

With all the aforementioned pieces in place, we now report our first fully self-consistent, relativistic dynamical simulations of BHNS binaries. We are particularly interested in binaries that may potentially lead to a sizable accretion disk, so we focus on binaries in which the neutron star is tidally disrupted just before reaching the innermost stable circular orbit (ISCO) and plunging into the black hole. The binary separation $d_{\text {tid }}$ at which the neutron star will be tidally disrupted may be estimated from the following crude Newtonian argument. Equating the tidal force exerted by the $\mathrm{BH}$ on a test mass at the NS's surface with the gravitational force exerted by the neutron star on this test mass, we find

$$
\frac{d_{\mathrm{tid}}}{M_{\mathrm{BH}}} \simeq q^{-2 / 3} \mathcal{C}^{-1}
$$

where $\mathcal{C} \equiv M_{\mathrm{NS}} / R_{\mathrm{NS}}$ is the neutron star compaction. Given typical neutron star compactions of $\mathcal{C} \sim 0.2$, small but reasonable values of $q$ are required for $d_{\text {tid }}$ to be larger than the ISCO separation of about $d_{\mathrm{ISCO}} \sim 6 M_{\mathrm{BH}}$. Our more careful analysis (36]; see Fig. 15) shows that for $\Gamma=2$ polytropes we need $q \lesssim 4.25$. Given typical neutron star masses $\left(M_{\mathrm{NS}} \sim 1.5 M_{\odot}\right)$, this means that we can expect the formation of an accretion disk only for low-mass black holes.

How often such binaries merge in the observable universe is still an open question. The uncertainties arise from some aspects of population synthesis calculations that are only partially understood. In particular, envelope ejection efficiency during the common envelope phase seems to be a crucial factor in forming low-mass BHs during binary stellar evolution. For example, if one assumes efficient ejection and a large maximum NS mass, the primary NS will generally accrete insufficient mass to induce collapse to a $\mathrm{BH}$, and one ends up with a large number of NSNS binaries. For inefficient ejection and a smaller maximum NS mass, it is relatively easy for the NS to accrete sufficient material to form a $\mathrm{BH}$ with a mass only slightly larger than a NS. Although some previous population synthesis calculations working under the latter assumption found a nearly flat spectrum of binary mass ratios spanning the range $q=1.5-10$ [69], a more recent calculation that assumes highly efficient envelope ejection yields typical binary mass ratios $q=6-10[70]$. The latter scenario would predict that NSs undergoing tidal breakup prior to reaching the ISCO are rare, as are any resulting SGRBs from these systems. The overall rate estimates for BHNS mergers observable by an advanced LIGO detector typically fall in the range $\mathcal{R} \sim 1-100 \mathrm{yr}^{-1}$ [71].

These issues noted, we begin our investigation of BHNS binary merger and coalescence in full general relativity. This paper is the first in a sequence of papers which will thoroughly explore the effect of various binary parameters on the tidal disruption, disk formation, the potential for launching a GRB, and the corresponding gravitational wave signals. In this paper we will focus on irrotational binaries with mass ratios $q \leq 3$. As an additional word of caution, we point out that our results are fundamentally limited by uncertainties about the true nuclear EOS, both in the cold initial state as well as the later shock-heated hot phase. Disk masses may depend sensitively on the structure of the NS, especially the lowdensity outer regions, so all BHNS merger results should be viewed in light of this caveat. In particular, the likelihood of BHNS mergers as SGRB progenitors may be difficult to determine conclusively until this issue is resolved.

This paper is organized as follows. In Secs. III and III. we summarize the basic equations and their specific implementation in our general relativistic hydrodynamics scheme, along with a discussion of initial data, gauge conditions, matter evolution, and diagnostics. In Sec. IV. we discuss the results of our BHNS merger simulations, and how they depend on both physical as well as computational parameter choices. We conclude in Sec. V with a discussion of our findings, and comment on future directions.

\section{BASIC EQUATIONS}

In this section we list the full set of evolution equations integrated by our numerical code. Field, coordinate, and hydrodynamic evolution equations are summarized in Secs. IIA, IB and IIC respectively.

\section{A. Field Evolution: The BSSN Equations}

Assuming geometrized units in which $G=c=1$, we write the spacetime metric in the standard $3+1$ form

$$
d s^{2}=-\alpha^{2} d t^{2}+\gamma_{i j}\left(d x^{i}+\beta^{i} d t\right)\left(d x^{j}+\beta^{j} d t\right),
$$


where $\alpha, \beta^{i}$, and $\gamma_{i j}$ are the lapse, shift, and spatial 3metric, respectively. The extrinsic curvature $K_{i j}$ is defined by

$$
\left(\partial_{t}-\mathcal{L}_{\beta}\right) \gamma_{i j}=-2 \alpha K_{i j} .
$$

Here $\mathcal{L}_{\beta}$ is the Lie derivative with respect to $\beta^{i}$.

In the BSSN formalism, we define the conformally related metric $\tilde{\gamma}_{i j}$, the conformal exponent $\phi$, the trace of the extrinsic curvature $K$, the conformal traceless extrinsic curvature $\tilde{A}_{i j}$, and the conformal connection functions $\tilde{\Gamma}^{i}$ as follows

$$
\begin{aligned}
\phi & =\frac{1}{12} \ln \left[\operatorname{det}\left(\gamma_{i j}\right)\right], \\
\tilde{\gamma}_{i j} & =e^{-4 \phi} \gamma_{i j}, \\
K & =\gamma_{i j} K^{i j} \\
\tilde{A}_{i j} & =e^{-4 \phi}\left(K_{i j}-\frac{1}{3} \gamma_{i j} K\right), \\
\tilde{\Gamma}^{i} & =-\tilde{\gamma}_{, j}^{i j},
\end{aligned}
$$

where ${ }_{j}$ denotes the partial derivative: $\tilde{\gamma}^{i j}{ }_{j} \equiv \partial_{j} \tilde{\gamma}^{i j}$. We use the same field evolution equations as Eqs. (11)-(15) of [72]:

$$
\begin{aligned}
\left(\partial_{t}-\mathcal{L}_{\beta}\right) \tilde{\gamma}_{i j}= & -2 \alpha \tilde{A}_{i j} \\
\left(\partial_{t}-\mathcal{L}_{\beta}\right) \phi= & -\frac{1}{6} \alpha K \\
\left(\partial_{t}-\mathcal{L}_{\beta}\right) K= & -\gamma^{i j} D_{j} D_{i} \alpha+\frac{1}{3} \alpha K^{2} \\
& +\alpha \tilde{A}_{i j} \tilde{A}^{i j}+4 \pi \alpha(\rho+S) \\
\left(\partial_{t}-\mathcal{L}_{\beta}\right) \tilde{A}_{i j}= & e^{-4 \phi}\left(-D_{i} D_{j} \alpha+\alpha\left(R_{i j}-8 \pi S_{i j}\right)\right)^{T F} \\
& +\alpha\left(K \tilde{A}_{i j}-2 \tilde{A}_{i l} \tilde{A}_{j}^{l}\right)
\end{aligned}
$$

and

$$
\begin{aligned}
\partial_{t} \tilde{\Gamma}^{i}= & \partial_{j}\left(2 \alpha \tilde{A}^{i j}+\mathcal{L}_{\beta} \tilde{\gamma}^{i j}\right) \\
= & \tilde{\gamma}^{j k} \beta^{i}{ }_{, j k}+\frac{1}{3} \tilde{\gamma}^{i j} \beta^{k}{ }_{, k j}-\tilde{\Gamma}^{j} \beta^{i}{ }_{, j} \\
& +\frac{2}{3} \tilde{\Gamma}^{i} \beta^{j}{ }_{, j}+\beta^{j} \tilde{\Gamma}^{i}{ }_{, j}-2 \tilde{A}^{i j} \partial_{j} \alpha \\
& -2 \alpha\left(\frac{2}{3} \tilde{\gamma}^{i j} K_{, j}-6 \tilde{A}^{i j} \phi_{, j}-\tilde{\Gamma}^{i}{ }_{j k} \tilde{A}^{j k}+8 \pi \tilde{\gamma}^{i j} S_{j}\right) .
\end{aligned}
$$

\section{B. Gauge Equations}

As in most moving puncture calculations, we use an advective " $1+\log$ " slicing condition for the lapse

$$
\partial_{t} \alpha-\beta^{i} \partial_{i} \alpha=2 \alpha K,
$$

and a second-order "non-shifting-shift" (in the language of 73$]$ )

$$
\begin{aligned}
\partial_{t} \beta^{i} & =\frac{3}{4} B^{i}, \\
\partial_{t} B^{i} & =\left(\frac{d r}{d \bar{r}}\right)^{2} \partial_{t} \tilde{\Gamma}^{i}-\eta B^{i} .
\end{aligned}
$$

This condition [37] is similar to that in [31], but allows for a fisheye radius $\bar{r}$, discussed in Sec. IIA below. This expression is closely related to the "Gamma-driver" family of shift evolution equations. We have found empirically that setting $\eta \sim 0.5 / M$ yields well-behaved coordinate evolutions, where $M$ is the ADM mass of the system, as defined in Eq. (37). This value is chosen for all runs except run $\mathrm{B}$, for which we use $\eta=0.413 / M$.

\section{Hydrodynamic Equations}

The matter source terms are defined as

$$
\begin{aligned}
\rho & =n_{\alpha} n_{\beta} T^{\alpha \beta} \\
S_{i} & =-\gamma_{i \alpha} n_{\beta} T^{\alpha \beta}, \\
S_{i j} & =\gamma_{i \alpha} \gamma_{j \beta} T^{\alpha \beta},
\end{aligned}
$$

where $T^{\alpha \beta} \equiv\left(\rho_{0}+\rho_{0} \epsilon+P\right) u^{\alpha} u^{\beta}+P g^{\alpha \beta}$ is the stressenergy tensor for a perfect fluid, $\rho_{0}, \epsilon, P$ and $u^{\alpha}$ are the fluid's rest-mass density, specific internal energy, pressure, and 4-velocity, respectively, and $n_{\alpha}=(-\alpha, 0,0,0)$ is the future-directed unit normal to the time slice.

We evolve the "conserved hydrodynamic variables", $\rho_{*}, \tilde{S}_{i}$ and $\tilde{\tau}$, defined as follows

$$
\begin{aligned}
\rho_{*} & =-n_{\mu} \rho_{0} u^{\mu}=\alpha \sqrt{\gamma} \rho_{0} u^{0}, \\
\tilde{S}_{i} & =\sqrt{\gamma} S_{i}=\alpha \sqrt{\gamma} T^{0}{ }_{i}=\rho_{*} h u_{i}, \\
\tilde{\tau} & =\sqrt{\gamma} n_{\mu} n_{\nu} T^{\mu \nu}-\rho_{*}=\alpha^{2} \sqrt{\gamma} T^{00}-\rho_{*} .
\end{aligned}
$$

The evolution equations for these variables are given by Eqs. (34), (36), and (38) of [66],

$$
\begin{aligned}
\partial_{t} \rho_{*}+\partial_{j}\left(\rho_{*} v^{j}\right) & =0 \\
\partial_{t} \tilde{S}_{i}+\partial_{j}\left(\alpha \sqrt{\gamma} T^{j}{ }_{i}\right) & =\frac{1}{2} \alpha \sqrt{\gamma} T^{\alpha \beta} \partial_{i} g_{\alpha \beta}, \\
\partial_{t} \tilde{\tau}+\partial_{i}\left(\alpha^{2} \sqrt{\gamma} T^{0 i}-\rho_{*} v^{i}\right) & =s
\end{aligned}
$$

where $\gamma \equiv \operatorname{det}\left(\gamma_{i j}\right)=e^{12 \phi}$, and the energy source term $s$ is

$$
\begin{aligned}
s= & -\alpha \sqrt{\gamma} T^{\mu \nu} \nabla_{\nu} n_{\mu} \\
= & \alpha \sqrt{\gamma}\left[\left(T^{00} \beta^{i} \beta^{j}+2 T^{0 i} \beta^{j}+T^{i j}\right) K_{i j}\right. \\
& \left.-\left(T^{00} \beta^{i}+T^{0 i}\right) \partial_{i} \alpha\right] .
\end{aligned}
$$

Here $v^{i} \equiv u^{i} / u^{0}$ is the fluid's 3 -velocity.

To complete the system of equations, we specify an EOS. Our code is capable of handling EOSs of the form $P=P\left(\rho_{0}, \epsilon\right)$. In this paper, we employ the standard $\Gamma$-law EOS

$$
P=(\Gamma-1) \rho_{0} \epsilon
$$

with $\Gamma=2$ to model the NS matter. 


\section{NUMERICAL METHODS}

The code we use is very similar to that described in [37, 38]. We do not consider magnetic fields in this paper, so the magnetic field sector is disabled in these calculations. The equations of general relativistic (GR) hydrodynamics are handled by a HRSC technique [66] that employs the monotonized central (MC) reconstruction scheme 74] coupled to the HLL (Harten, Lax, and van Leer) approximate Riemann solver 75]. The metric is evolved via the BSSN formalism [58, 59] as described in [72], but with fourth-order accurate spatial differencing and upwinding on the shift advection terms. Our code is based on the Cactus parallelization framework [76], in which our second-order Iterated Crank-Nicholson time-stepping is managed by the MoL, or method of lines, thorn.

For completeness, we provide below a brief overview of our grid setup and initial data (Sec. IIIA); a discussion of field, gauge (Sec. IIIB), and hydrodynamic (Sec. IIC) evolution techniques; a description of how we apply boundary conditions (Sec. IIID); a summary of diagnostic techniques we use to both validate our numerical results and examine our spacetimes (Sec. IIIE); and finally a description of the technique we use to measure gravitational wave (GW) emission (Sec. ПIIF).

\section{A. Grid Setup and Initial data}

We use a "fisheye" coordinate system 77] to expand the physical extent of our numerical grid while maintaining high resolution in the strong-field region. Significantly lower resolution is maintained in the wavezone, but it is set so that a gravitational wavelength is resolved by at least 12 gridpoints. To set up a fisheye coordinate grid, we define the "physical" radius $r$ in terms of a fisheye radius $\bar{r}$ according to

$$
r=a_{n} \bar{r}+\sum_{i=1}^{n} \frac{\left(a_{i-1}-a_{i}\right) s_{i}}{2 \tanh \left(R_{i} / s_{i}\right)} \ln \left(\frac{\cosh \left(\left(\bar{r}+R_{i}\right) / s_{i}\right)}{\cosh \left(\left(\bar{r}-R_{i}\right) / s_{i}\right)}\right) .
$$

Here $a_{i}$ sets the magnitude of the $i$ 'th fisheye transition, $s_{i}$ determines the width of the transition, and $R_{i}$ specifies the center of the transition. In physical coordinates, the grid spacing smoothly transforms from $\Delta x \approx a_{i-1} \Delta \bar{x}$ to $\Delta x \approx a_{i} \Delta \bar{x}$, over a set of fisheye coordinates spanning radii $R_{i}-s_{i}<\bar{r}<R_{i}+s_{i}$. For convenience, we always set $a_{0}=1$, so that our coordinate grid spacing $\Delta x$ represents the physical grid spacing in the central region of our numerical grid. In this paper we use only one transition zone $(n=1)$, with $a_{1}=8$. We have listed other relevant grid parameters in Table II.

We defer to Appendix A of [37] for transformation laws pertaining to all field and hydrodynamic quantities under a fisheye transformation. All of our calculations are performed assuming equatorial symmetry, on numerical grids of the form $2 N \times 2 N \times N$, with $N$ ranging from 166 to 305 .

All initial data we evolve in this paper were generated by 35]. To map these spectral configurations onto our non-spectral simulation grid, we first construct our numerical grid and record the positions of each point in physical coordinates. Then we evaluate the field and hydrodynamic quantities based on their spectral coefficients. Next, we transform the vector and tensor quantities into fisheye coordinates via transformations found in Appendix A of [37]. Finally, the excised $\mathrm{BH}$ region is filled with constraint-violating initial data, using the "smooth junk" technique we developed and validated in [38.

The assumptions under which our initial data are constructed differ from those of SU and ST. We solve Einstein's constraint equations in the conformal thinsandwich (CTS) formalism, which allows us to impose an approximate helical Killing vector by setting the time derivatives of the conformally related metric to zero. As a result, our extrinsic curvature is always related to the shift vector that appears in the solution through Eq. (4) of [36],

$$
\tilde{A}^{i j} \equiv \frac{\psi^{6}}{2 \alpha}\left(\nabla^{i} \beta^{j}+\nabla^{j} \beta^{i}-\frac{2}{3} \tilde{\gamma}^{i j} \nabla_{k} \beta^{k}\right) .
$$

These quasiequilibrium initial data excise the black hole interior, allowing us to impose equilibrium boundary conditions on the excision surface. By contrast, SU and ST adopt the CTT decomposition to obtain the extrinsic curvature on the initial slice, but employ the assumption of a helical Killing vector to construct a lapse and shift. Also, they model the black hole as a puncture (see [43, 44, 45, 46]). Both sets of initial data are solutions to Einstein's constraint equations, but they may differ in both the amount of spurious gravitational wave content and the degree of orbital eccentricity.

\section{B. Metric Evolution and Gauge}

We apply two methods that have improved the stability and accuracy of our field and gauge evolution when evolving $\mathrm{BH}$ spacetimes.

First, we use fourth-order finite differencing schemes to calculate spatial derivatives in the field/gauge evolution sectors. Also, for any terms of the form $\beta^{i} \partial_{i} \ldots$, which arise in both the Lie derivative terms and in the lapse evolution, we use fourth order upwind differencing stencils instead of the standard centered fourth-order stencils (see Eqs. (2.5)-(2.6) and (2.2)-(2.4) of [78], respectively). We note for completeness that our mixed second-derivative stencil is slightly different that given by Eq.(2.4) of [78], but remains fourth-order convergent,

$$
\begin{aligned}
\partial_{x y} F_{i, j, k}= & \frac{1}{48 d x d y}\left[F_{i-2, j+2, k}+F_{i+2, j-2, k}\right. \\
& -F_{i+2, j+2, k}-F_{i-2, j-2, k}
\end{aligned}
$$




$$
\begin{aligned}
& +16\left(F_{i+1, j+1, k}+F_{i-1, j-1, k}\right. \\
& \left.\left.-F_{i-1, j+1, k}-F_{i+1, j-1, k}\right)\right] .
\end{aligned}
$$

In addition, we enforce the conditions $\tilde{\gamma} \equiv \operatorname{det}\left(\tilde{\gamma_{i j}}\right)=$ 1 and $\tilde{A} \equiv \operatorname{tr}\left(\tilde{A}_{i j}\right)=0$ at every timestep, using the substitutions

$$
\begin{aligned}
& \tilde{\gamma}_{i j} \rightarrow \tilde{\gamma}_{i j} / \tilde{\gamma}^{1 / 3}, \\
& \tilde{A}_{i j} \rightarrow \tilde{A}_{i j}-\frac{\tilde{A}}{3} \tilde{\gamma}_{i j},
\end{aligned}
$$

as is commonly done in numerical relativity codes. We do not, however, enforce the Hamiltonian, momentum, or Gamma constraints,

$$
\begin{aligned}
0=\mathcal{H}= & \tilde{\gamma}^{i j} \tilde{D}_{i} \tilde{D}_{j} e^{\phi}-\frac{e^{\phi}}{8} \tilde{R} \\
& +\frac{e^{5 \phi}}{8} \tilde{A}_{i j} \tilde{A}^{i j}-\frac{e^{5 \phi}}{12} K^{2}+2 \pi e^{5 \phi} \rho, \\
0=\mathcal{M}^{i}= & \tilde{D}_{j}\left(e^{6 \phi} \tilde{A}^{j i}\right)-\frac{2}{3} e^{6 \phi} \tilde{D}^{i} K-8 \pi e^{6 \phi} S^{i}, \\
0=\mathcal{G}^{i}= & \tilde{\Gamma}^{i}+\tilde{\gamma}^{i j}{ }_{j},
\end{aligned}
$$

so these serve as an independent check on the validity of our code. We do not add a Hamiltonian constraint damping term to the right hand side (RHS) of the $\phi$ evolution equation, but we add damping terms to the RHS of the $\tilde{\Gamma}^{i}, \tilde{\gamma}_{i j}$, and $\tilde{A}_{i j}$ BSSN evolution equations, following the prescription defined in [79]. These terms are zero analytically, and serve only to stabilize evolutions.

\section{Hydrodynamic evolution}

The hydrodynamics equations are calculated using the HRSC scheme described by [66]. To recover the "primitive variables" $\rho_{0}, P$, and $v^{i}$ from the conserved set $\rho_{*}, \tilde{\tau}$, and $\tilde{S}_{i}$, we perform the inversion as specified by Eqs. (57)-(62) of [66]. As in [37], our inversion algorithm occasionally finds unphysical sets of conserved variables at points immediately adjacent to the puncture and in our atmosphere, which do not allow for solutions of the primitive variables. As in that paper, we enforce the following two conditions, which are both necessary and sufficient to allow for a well-defined inversion everywhere, and result in smooth hydrodynamic variable profiles in the $\mathrm{BH}$ interior after the puncture has passed through a set of grid points:

$$
\begin{aligned}
|\tilde{S}|^{2} \equiv \gamma^{i j} \tilde{S}_{i} \tilde{S}_{j} & <\tilde{\tau}\left(\tilde{\tau}+2 \rho_{*}\right), \\
\tilde{\tau} & >0 .
\end{aligned}
$$

When these conditions are not met we rescale $\tilde{S}^{i}$ so that its new magnigude is $|\tilde{S}|^{2}=0.98 \tilde{\tau}\left(\tilde{\tau}+2 \rho_{*}\right)$, and set $\tilde{\tau}=$ $10^{-18} \tilde{\tau}_{0 ; \text { max }}$, where $\tilde{\tau}_{0 ; \text { max }}$ is the maximum value of $\tilde{\tau}$ present in our initial data.

To stabilize our hydrodynamic scheme in regions where there is no matter, we maintain a tenuous atmosphere on our grid, with a density floor set to $10^{-10}$ of the maximum density on our grid at $t=0$. The initial atmospheric pressure $P_{\text {atm }}$ is set to the cold polytropic value $P_{\text {atm }}=\kappa \rho_{\text {atm }}^{\Gamma}$, where $\kappa$ is the polytropic constant at $t=0$. Throughout the evolution, we impose limits on the atmospheric pressure to prevent spurious heating and negative values of the internal energy $\epsilon$.

\section{Boundary Conditions}

We apply Sommerfeld outgoing wave boundary conditions to the entire set of field and gauge variables $\mathbf{f}$

$$
\mathbf{f}(r, t)=\frac{r-\Delta r}{r} \mathbf{f}(r-\Delta r, t-\Delta T)
$$

on the outer boundary of our numerical grid. Here $\Delta T$ is the timestep and $\Delta r=\alpha e^{-2 \phi} \Delta T$, where radii are evaluated in physical (as opposed to fisheye) coordinates. To enforce these boundary conditions, we first transform $\beta^{i}$, $\phi, \tilde{A}_{i j}$, and $\tilde{g}_{i j}$ into physical coordinates, apply the Sommerfeld condition as specified above, and then transform back to fisheye coordinates. We do not transform $\tilde{\Gamma}^{i}$, assuming that its value propagates outward independent of the radius.

Since we adopt the moving puncture method (as opposed to black hole excision) there are no interior boundaries or boundary conditions.

\section{E. Diagnostics}

To validate our calculations, we compute surface integrals for the system ADM mass $M$, linear momentum $P_{i}$, and angular momentum $J_{i}$, given by

$$
\begin{aligned}
M & =\frac{1}{2 \pi} \oint\left(\frac{1}{8} \tilde{\Gamma}^{i}-\tilde{\gamma}^{i j} \partial_{j} \psi\right) d \Sigma_{i}, \\
P_{i} & =\frac{1}{8 \pi} \oint\left(K_{i}^{j}-\delta_{i}^{j} K\right) d \Sigma_{j}, \\
J_{i} & =\frac{1}{8 \pi} \epsilon_{i j}^{k} \oint x^{j}\left(K_{k}^{m}-\delta_{k}^{m} K\right) d \Sigma_{m},
\end{aligned}
$$

where $\psi=e^{\phi}$ and $d \Sigma_{i}=\left(x^{i} / r\right) \psi^{6} r^{2} \sin \theta d \theta d \varphi$ for a spherical surface at fixed radius. Note that the above expressions are valid only if the spatial 3-metric $\gamma_{i j}$ approaches the Minkowski metric $\eta_{i j}$ at large $r$. Hence we need to transform the variables from fisheye to physical coordinates before integrating. In addition, we monitor the following normalized expressions for the Hamiltonian and momentum constraints

$$
\begin{aligned}
\|\mathcal{H}\| & \equiv \int_{\mathcal{V}}\left(|\mathcal{H}| / N_{\mathrm{HC}}\right) d V \\
\left\|\mathcal{M}^{i}\right\| & \equiv \int_{\mathcal{V}}\left(\left|\mathcal{M}^{i}\right| / N_{\mathrm{MC}}\right) d V
\end{aligned}
$$


where

$$
\begin{aligned}
N_{\mathrm{HC}}= & \left(\left(2 \pi \psi^{5} \rho\right)^{2}+\left(\tilde{D}^{i} \tilde{D}_{i} \psi\right)^{2}+\left(\frac{\psi}{8} \tilde{R}\right)^{2}\right. \\
& \left.+\left(\frac{\psi^{5}}{8} \tilde{A}_{i j} \tilde{A}^{i j}\right)^{2}+\left(\frac{\psi^{5}}{12} K^{2}\right)^{2}\right)^{1 / 2}, \\
N_{\mathrm{MC}}= & \left(\sum _ { i = 1 } ^ { 3 } \left[\left(8 \pi S^{i}\right)^{2}+\left(\frac{2}{3} \tilde{D}^{i} K\right)^{2}\right.\right. \\
& \left.\left.+\left(\psi^{-6} \tilde{D}_{j}\left(\psi^{6} \tilde{A}^{i j}\right)\right)^{2}\right]\right)^{1 / 2} .
\end{aligned}
$$

The apparent horizon $(\mathrm{AH})$ of the $\mathrm{BH}$ is computed using the ahfinderdirect Cactus thorn [80]. This thorn outputs the BH irreducible mass, which is related to the $\mathrm{AH}$ area $\mathcal{A}$ as follows:

$$
M_{\mathrm{irr}}=\sqrt{\mathcal{A} / 16 \pi} .
$$

With the AH surface computed by ahfinderdirect, we can evaluate diagnostic integrals in a region interior or exterior to the $\mathrm{AH}$.

\section{F. Gravitational radiation}

To measure the gravitational wave (GW) emission from our binaries, we use both gauge-invariant theory based on perturbations of a background Schwarzschild spacetime derived by Zerilli [81] and Moncrief [82], hereafter referred to as the "Z-M" formalism, as well as a technique that makes use of the Newman-Penrose Weyl scalar $\psi_{4}$. In the Z-M formulation, deformations of the spatial metric are viewed as perturbations on a Schwarzschild background at large radii. We decompose these perturbations into gauge-independent even and odd-parity modes, denoted $\Psi_{\text {even }}^{l m}$ and $Q_{\mathrm{M}}^{l m}$ in the notation of [83], whose derivation is outlined below. For convenience, we define the time-integral of the odd-parity mode amplitude

$$
\Psi_{o d d}^{l m} \equiv-\int_{-\infty}^{t} Q_{\mathrm{M}}^{l m} d t^{\prime} .
$$

In terms of these expressions, the complex gravitational wave strain $H \equiv h_{+}-i h_{\times}$is given by Eq. (4.34) of [83],

$$
H=\frac{1}{2 r} \sum_{l, m} \sqrt{\frac{(l+2) !}{(l-2) !}}\left(\Psi_{\text {even }}^{l m}+i \Psi_{\text {odd }}^{l m}\right)_{-2} Y^{l m},
$$

where ${ }_{-2} Y^{l m}$ is the $s=-2$ spin-weighted spherical harmonic. In the notation of Shibata and collaborators (see, e.g., [84] and earlier papers), $R_{l m}^{\mathrm{E}}$ and $R_{l m}^{\mathrm{O}}$ are related to our adopted notation as follows:

$$
R_{l m}^{\mathrm{E}} \equiv \frac{1}{\sqrt{2} r} \sqrt{\frac{(l+2) !}{(l-2) !}} \Psi_{e v e n}^{l m}
$$

$$
R_{l m}^{\mathrm{O}} \equiv \frac{1}{\sqrt{2} r} \sqrt{\frac{(l+2) !}{(l-2) !}} \Psi_{o d d}^{l m} .
$$

In addition, we use the PsiKadelia thorn to compute the complex Weyl scalar $\psi_{4}$, which depends on the spatial metric and extrinsic curvature. The wave strains are given in terms of $\psi_{4}$ by Eq. (3.4) of [83],

$$
H=-\int_{-\infty}^{t} \int_{-\infty}^{t^{\prime}} \psi_{4} d t^{\prime} d t^{\prime \prime}
$$

We also decompose $\psi_{4}$ into $s=-2$ spin-weighted spherical harmonic modes.

In terms of $H$, the radiated energy, linear momentum, and z-component of the angular momentum are calculated from Eqs. (2.8), (2.11), and (2.13) of [83]

$$
\begin{aligned}
\frac{d E}{d t} & =\lim _{r \rightarrow \infty} \frac{r^{2}}{16 \pi} \oint|\dot{H}|^{2} d \Omega, \\
\frac{d P^{i}}{d t} & =\lim _{r \rightarrow \infty} \frac{r^{2}}{16 \pi} \oint \frac{x^{i}}{r}|\dot{H}|^{2} d \Omega, \\
\frac{d J_{z}}{d t} & =-\lim _{r \rightarrow \infty} \frac{r^{2}}{32 \pi} \oint H^{*} \partial_{\phi} H d \Omega .
\end{aligned}
$$

In practice, radiative losses are computed as a sum over modes (up to and including $l=4$ ), following expressions equivalent to Eqs. (4.41), (4.43), and (4.47) of [83] for the Z-M case, and Eqs. (3.6), (3.8), (3.14), and (3.24) for the $\psi_{4}$ case.

To compute the gravitational wave energy spectra, we use the same techniques as 14], determining the energy loss per unit frequency from their Eq. (8),

$$
\frac{d E}{d f}=\frac{\pi}{8} \sum_{l, m} f^{2} \frac{(l+2) !}{(l-2) !}\left|\tilde{\Psi}_{l m}\right|^{2}
$$

where $\left|\tilde{\Psi}_{l m}\right| \equiv\left|\tilde{\Psi}_{\text {even }}^{l m}\right|^{2}+\left|\tilde{\Psi}_{\text {odd }}^{l m}\right|^{2}$, and $\tilde{\Psi} \equiv$ $\int e^{2 \pi i f t} \Psi(t) d t$. We also define the effective gravitational wave amplitude, taking the $z \rightarrow 0$ limit of Eq. (5.1) from [85]:

$$
h_{\mathrm{eff}}(f) \equiv \frac{\sqrt{2}}{\pi r} \sqrt{\frac{d E}{d f}} .
$$

Notice that this expression differs by a proportionality constant from that found in [86]. This is due to different assumptions regarding the alignment of the binary and viewing angle relative to the detectors. The constant here is specified to reflect the RMS average of signal amplitudes over all possible orientations of merging binaries and the detector.

\section{NUMERICAL CALCULATIONS OF BHNS MERGER}

To begin our survey of the BHNS merger parameter space, we focus on those configurations likely to undergo 
mass loss and disk formation, i.e., systems with binary mass ratios comparable to unity. To ensure that our results are physically relevant, we consider cases with appropriately large NS compactions, noting that lowering the compactions would certainly increase the mass of a potential disk.

All our calculations begin with initial configurations taken from 35], which consist of irrotational NSs orbiting approximately nonspinning BHs. We investigate two different cases for the NS compaction, distinguished by nondimensional mass $\bar{M}_{0} \equiv M_{0} / \kappa^{1 / 2}=0.15$ and 0.1 , where $M_{0}$ is the rest (baryon) mass of the $\mathrm{NS}$, and $\kappa$ is the polytropic constant. We refer to these as the high and low-compaction NS cases. These configurations yield compactions $\mathcal{C}=M_{\mathrm{NS}} / R_{\mathrm{NS}}$ of 0.145 and 0.0879 , respectively, where $M_{\mathrm{NS}}$ is the ADM mass and $R_{\mathrm{NS}}$ is the radius of the NS in isolation. Setting $\kappa$ fixes the NS mass for a given compaction and polytropic index [87]. Choosing the high (low) compaction NS to have a rest mass of $1.4 M_{\odot}$, we find the ADM mass for the isolated NS to be $1.30 M_{\odot}\left(1.34 M_{\odot}\right)$, with an isotropic radius of $11.24 \mathrm{~km}$ $(20.46 \mathrm{~km})$, and circumferential (Schwarzschild) radius of $13.24 \mathrm{~km}$ and $(22.49 \mathrm{~km})$.

We consider data from four different independent sequences in [35], consisting of the high-compaction NS in binaries with mass ratios $q \equiv M_{\mathrm{irr}} / M_{N S}=3$, 2, and 1 , as described in their Table IV, along with the lowcompaction NS for a binary mass ratio $q=2$, from their Table V. Here, $M_{\text {irr }}$ is the BH irreducible mass. These sequences are denoted "A"- "D", respectively, and are summarized in Table 1 .

For sequence A, we consider three separate initial binary separations (denoted A1, A2, and A3) to determine how the initial separation affects the results of the simulations. For case A1, we performed a series of simulations designed to study the effect of the numerical grid parameters on our results. In cases A1-lo, A1-med, and A1-hi, we varied only the grid resolution, keeping the outer boundary at a fixed location. Case A1-farbc has the same grid resolution as A1-med, but the outer boundary is farther away.

Performing multiple runs with the same quasiequilibrium binary parameters (sequence A) enables us to gauge the resources necessary to perform accurate evolutions and validate our physical results. For example, we may compare the location of the tidal disruption point against quasiequilibrium estimates of [36]. We also investigate how the disk mass varies with regard to our grid parameters, noting that this had a significant effect on the results found in [19, 20]. Finally, performing multiple runs allows us to check the robustness of the gravitational wave signals.

For convenience, we identify the approximate time at which the low-density regions of the deformed NS first fall into the BH horizon as the moment of "first contact", $t_{\mathrm{FC}}$.

\section{A. BHNS binaries with mass ratio $3: 1$}

To begin our discussion, we consider results from evolutions of sequence A, the high compaction NS case with $\bar{M}_{0}=0.15$ and $q=3$.

In Fig. 1, we plot density contours with overlaid 3velocity vectors in the equatorial plane for our large initial separation case (A3). The orbital direction is counter-clockwise in these snapshots. The upper left panel shows the initial configuration. We see the onset of accretion after approximately 1.75 orbits $(t \approx 215 M)$, with matter flowing in a narrow stream through the inner Lagrange point and into the BH. The accretion flow then accelerates as the NS is consumed by the BH. Later, at $t \approx 290 M$, we see the beginnings of mass loss outward into a disk through the outer Lagrange point, but the mass stream is quite tenuous. At the end of our simulation $(t=418.7 M)$, no more than $3 \%$ of the total rest mass of the NS exists outside the AH. The remaining matter is gravitationally bound to the BH. However, not all of this matter will form what would typically be referred to as a disk, i.e., a quasi-stationary torus that evolves on secular rather than dynamical timescales. Instead, some of the exterior mass at the end of our calculations will be accreted on relatively short timescales, until the remaining fraction achieves rotationally-supported quasi-equilibrium. Thus, these estimates should be taken as upper limits on true "disk masses" for a particular set of initial data and grid parameters.

In case A of [19], SU perform a fully GR simulation of a BHNS merger with a synchronized NS and a mass ratio of $q=2.47$. They find the fraction of the initial rest mass of the NS in the final disk to be $\approx 19-28 \%$ of the initial rest mass of the NS. We find this disk mass fraction to be $\lesssim 3 \%$ for our $q=3$, irrotational NS model A3, which suggests that more slowly spinning NSs feed less mass into a disk. In case A of [21], ST simulate an irrotational, $q=3.06$ BHNS binary and obtain a disk with a rest mass that is $\approx 6.6 \%$ of the initial NS rest mass. While this result is consistent with our observation that lower NS spin suppresses the mass of the disk, ST still find a significantly larger disk than we do.

To check the validity of our simulations, we monitor the normalized Hamiltonian and momentum constraint violations using Eqs. (40) and (41), respectively. We show the results from sequence A in Fig. 2, In all cases, the Hamiltonian and momentum constraint violations are $\sim 3 \%$ throughout the evolution. We find that at early times, the Hamiltonian constraint violation decreases as resolution is increased, as expected. However, at late times the Hamiltonian constraint violation steadily increases, and its magnitude becomes insensitive to resolution (see lines corresponding to cases A1-hi, A1-med and A1-low in Fig. 2), suggesting that the error is dominated by reflection from the outer boundaries. Indeed, we find that the Hamiltonian constraint violation is significantly smaller when we move the outer boundaries outward (see case A1-farbc in Fig. 2). 
TABLE I: Summary of our initial configurations, which are taken from the results of [35]. Here, the mass ratio $q \equiv M_{\text {irr }} / M_{N S}$, $\bar{M}_{0} \equiv M_{0} / \kappa^{1 / 2}$ is the nondimensional rest (baryon) mass, $M$ is the total ADM mass for the binary system (assuming that $\left.M_{0}=1.4 M_{\odot}\right), a_{0}$ the initial binary coordinate separation, $J$ the initial angular momentum of the system, $\Omega$ the orbital frequency, and $t_{\mathrm{FC}}$ the time of first contact. Finally, $M \Omega_{\mathrm{CTS}}$ is the frequency of tidal disruption, as derived from our initial data [35], and $M \Omega_{\text {num }}$ is the frequency at which our numerically derived waveform spectrum deviates from the restricted post-Newtonian value by $25 \%$ (see Section IV C).

\begin{tabular}{|cccccccccc|}
\hline Case & $q$ & $\bar{M}_{0}$ & $M / M_{\odot}$ & $a_{0} / M$ & $J / M^{2}$ & $M \Omega$ & $t_{\mathrm{FC}} / M$ & $M \Omega_{\mathrm{CTS}}$ & $M \Omega_{\text {num }}$ \\
\hline $\mathrm{A} 1$ & 3.0 & 0.15 & 5.15 & 5.41 & 0.629 & 0.0628 & 50 & 0.0728 & - \\
$\mathrm{A} 2$ & 3.0 & 0.15 & 5.16 & 6.49 & 0.663 & 0.0435 & 105 & 0.0728 & - \\
$\mathrm{A} 3$ & 3.0 & 0.15 & 5.17 & 8.81 & 0.698 & 0.0329 & 215 & 0.0728 & 0.0789 \\
$\mathrm{~B}$ & 2.0 & 0.15 & 3.86 & 7.17 & 0.774 & 0.0499 & 60 & 0.0550 & 0.0656 \\
$\mathrm{C}$ & 1.0 & 0.15 & 2.57 & 8.61 & 0.936 & 0.0343 & 91 & 0.0382 & 0.0509 \\
$\mathrm{D}$ & 2.0 & 0.1 & 3.98 & 11.56 & 0.909 & 0.0228 & 155 & 0.0255 & 0.0395 \\
\hline
\end{tabular}

TABLE II: Summary of the grid configurations and fisheye parameters used for our runs.

\begin{tabular}{|ccccccc|}
\hline Run & $\Delta x_{\text {int }} / M$ & $\Delta x_{\text {ext }} / M$ & Grid Size & $r_{\text {out }} / M$ & $R_{1} / M$ & $s_{1} / M$ \\
\hline A1-hi & 0.05 & 0.4 & $540^{2} \times 270$ & 39.5 & 9.7 & 1.6 \\
A1-med & 0.061 & 0.49 & $440^{2} \times 220$ & 39.5 & 9.7 & 1.6 \\
A1-lo & 0.081 & 0.65 & $332^{2} \times 166$ & 39.5 & 9.7 & 1.6 \\
A1-farbc & 0.061 & 0.49 & $604^{2} \times 302$ & 79.6 & 9.7 & 1.6 \\
A2 & 0.061 & 0.49 & $532^{2} \times 266$ & 39.7 & 12.9 & 1.6 \\
A3 & 0.061 & 0.49 & $516^{2} \times 258$ & 40.0 & 12.3 & 1.6 \\
B & 0.081 & 0.65 & $404^{2} \times 202$ & 50.2 & 11.6 & 2.2 \\
C & 0.05 & 0.4 & $610^{2} \times 305$ & 40.0 & 11.7 & 1.6 \\
D & 0.061 & 0.49 & $566^{2} \times 283$ & 40.1 & 15.8 & 1.6 \\
\hline
\end{tabular}

Next, we investigate how our results depend on both the initial binary separation and the numerical resolution. In Fig. 3, we plot the fraction of the rest (baryon) mass inside the apparent horizon, $f_{\text {in }} \equiv M_{0}(r<$ $\left.r_{\mathrm{AH}}\right) / M_{0}$, as a function of time for sequence $\mathrm{A}$. We find that $f_{\text {in }}$ depends only very weakly on the resolution or the location of the outer boundaries. We do, however, find stronger dependence on the initial binary separation (column $a_{0} / M$ in Table【). This dependence may be due to the zero radial infall speed in our initial data, which results in a slightly eccentric orbit that increases the radial infall speed as the binary approaches the ISCO, affecting the tidal disruption and the disk formation (compare [88, 89, 90, 91]). This effect may be compounded by the fact that our initial configuration is very close to the ISCO. Alternatively, this dependence may be caused by growing numerical error during the simulation. Despite these small uncertainties, we find $f_{\text {in }} \gtrsim 97 \%$ at the end of our simulations in all our cases, suggesting that there is no appreciable disk left behind after merger.

Figure 4 shows the evolution of the $\mathrm{BH}$ irreducible mass, $M_{\mathrm{irr}}$, for the sequence A1 simulations. We see that $M_{\text {irr }}$ increases as the NS matter is accreted, as expected. At late times, $M_{\text {irr }}$ approaches an asymptotic value when most of the matter has fallen into the $\mathrm{BH}$. However, reflection from the outer boundaries causes $M_{\text {irr }}$ to slowly increase at very late times for runs A1-hi, A1-med and A1-low. This spurious effect is significantly reduced when the outer boundaries are moved to a larger radius (run A1-farbc), and $M_{\text {irr }} \rightarrow 0.954 M$ at late times.

\section{B. The effect of the binary mass ratio and NS compaction}

Fig. 5 demonstrates the variation in dynamics due to a change in binary mass ratio, keeping the NS companion fixed. All three cases evolve approximately 0.5 orbits before the tidally disrupted NS first touches the apparent horizon (i.e, the time of "first contact" - the top 3 plots of Fig. (5). At this point in time, the general morphology of the systems are the same, regardless of mass ratio: a funnel-shaped NS, with matter flowing through the narrow end of the funnel into the BH. After the time of "first contact" $\left(t^{\prime} \equiv t-t_{\mathrm{FC}}>0\right)$, the dynamics of the system depend most sensitively on the initial mass, and hence size, of the black hole in comparison to the size of the NS. Defining $\chi_{L}^{\text {funnel }}$ as the (coordinate) angular extent of the accretion funnel (with the density cutoff as defined in Fig. 11) around the $\mathrm{AH}$ on the equatorial plane, we find that as $\chi_{L}^{\text {funnel }}$ increases to $180^{\circ}$, the accretion rate slows.

For example, in the A cases $(q=3)$, the $\mathrm{BH}$ is sufficiently large so that $\chi_{\llcorner}^{\text {funnel }}<180^{\circ}$ throughout much of the simulation. We see that the NS accretion rate does not slow down until $\approx 90 \%$ of the rest mass falls into the 

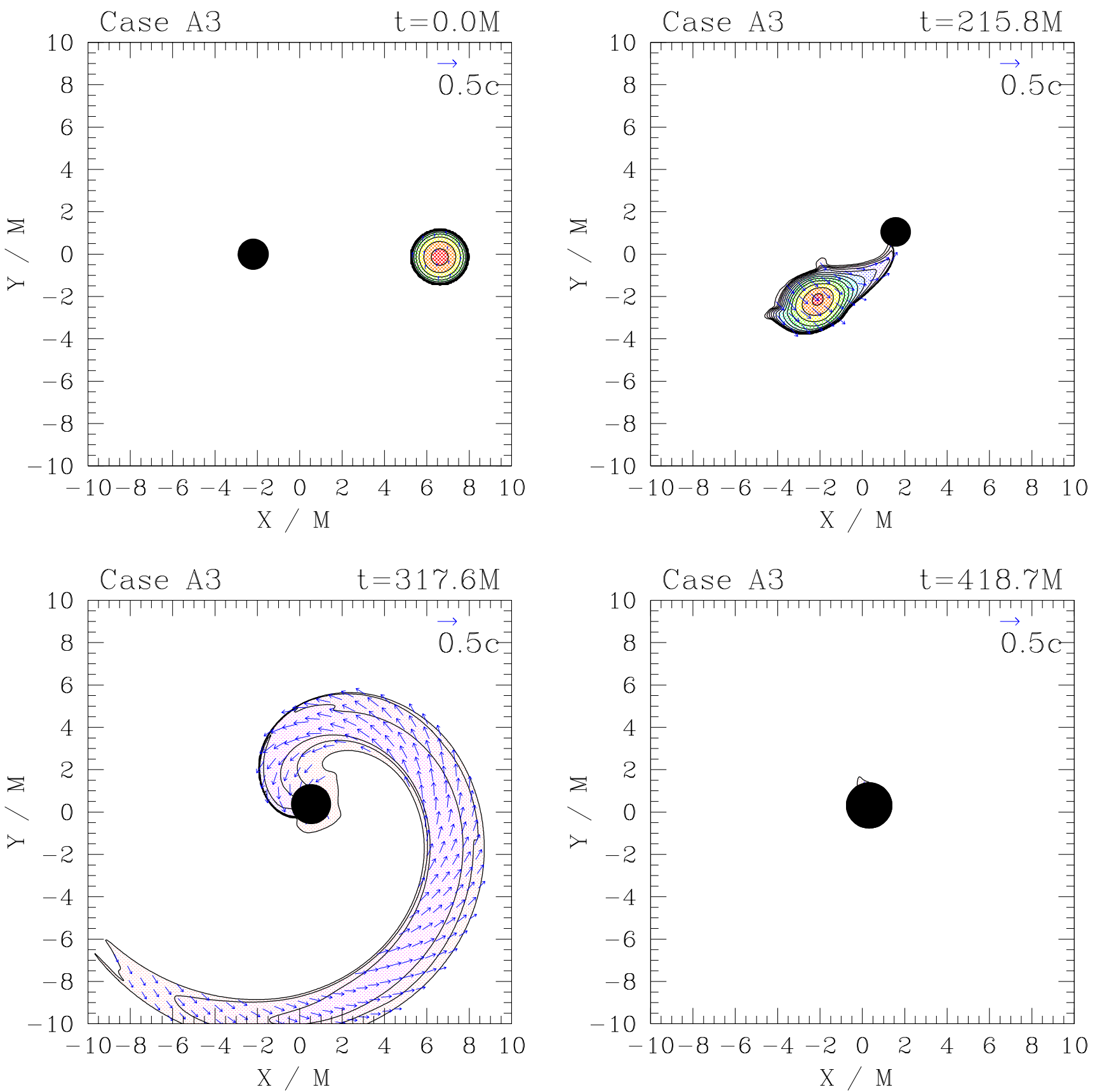

FIG. 1: Snapshots of density and velocity profiles at selected times for run A3, with binary mass ratio $q=3$. The contours represent the density in the orbital plane, plotted logarithmically with four contours per decade, with greyscaling added for clarity. Arrows represent the velocity field in the orbital plane. The minimum contour value in each frame is $\kappa \rho_{0}(\min )=10^{-4}$, or $\rho_{0}(\min )=7 \times 10^{11}\left(1.4 M_{\odot} / M_{0}\right)^{2} \mathrm{~g} \mathrm{~cm}^{-3}$. The maximum initial NS density is $\kappa \rho_{0}=0.13$. We specify the black hole AH interior in each snapshot with a filled black circle. In cgs units, the total ADM mass for this case is $M=3 \times 10^{-5}\left(M_{0} / 1.4 M_{\odot}\right)$ $\mathrm{s}=8\left(M_{0} / 1.4 M_{\odot}\right) \mathrm{km}$.

BH (Fig. 6). After the densest part of the NS falls into the $\mathrm{BH}\left(t^{\prime} \gtrsim 30 M\right)$, the remaining NS funnel curls and expands into a long, low-density tail while accretion continues. During the tail phase, the accretion rate slows until $\approx 99 \%$ of the NS rest mass is inside the $\mathrm{BH}$ (see
Figs. 5 and 6).

In case $\mathrm{B}(q=2)$, the $\mathrm{BH}$ is $33 \%$ smaller and $\chi_{L}^{\text {funnel }}$ reaches $\approx 180^{\circ}$ at $t^{\prime} \approx 40 \mathrm{M}$, at about the time when the densest part of the NS core falls into the BH. The remaining material in the funnel begins to evolve into a tail around this time, when only $\approx 55 \%$ of the NS rest mass 


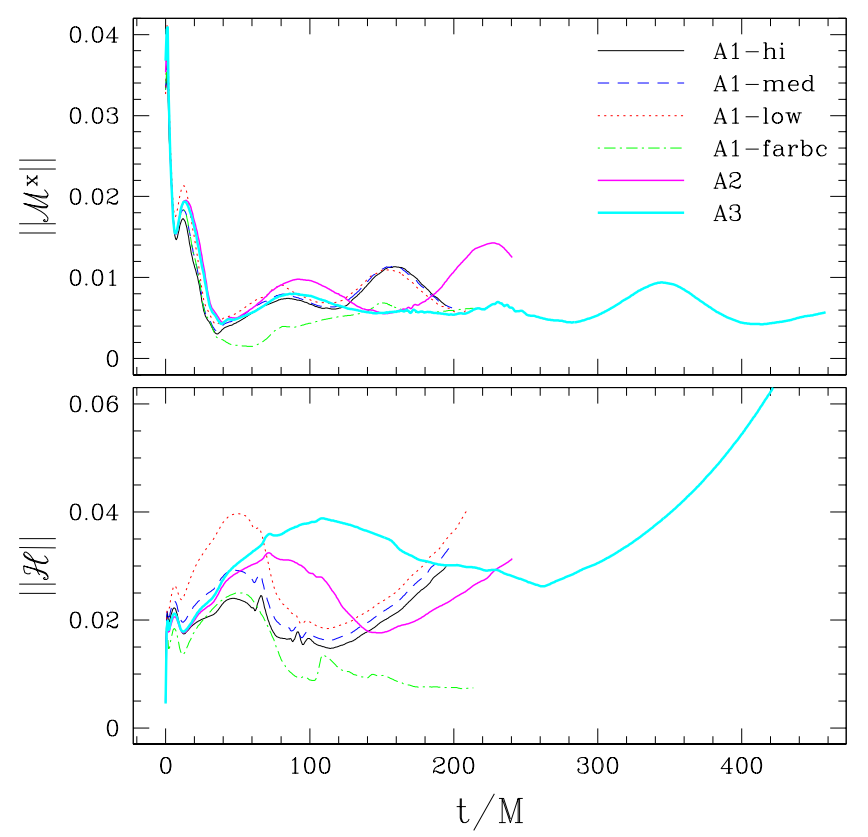

FIG. 2: Normalized violation of the $x$-component of the momentum constraint $\left\|\mathcal{M}^{x}\right\|$ (top panel) and the Hamiltonian constraint $\|\mathcal{H}\|$ (bottom panel); see Eqs. (40) and (41)

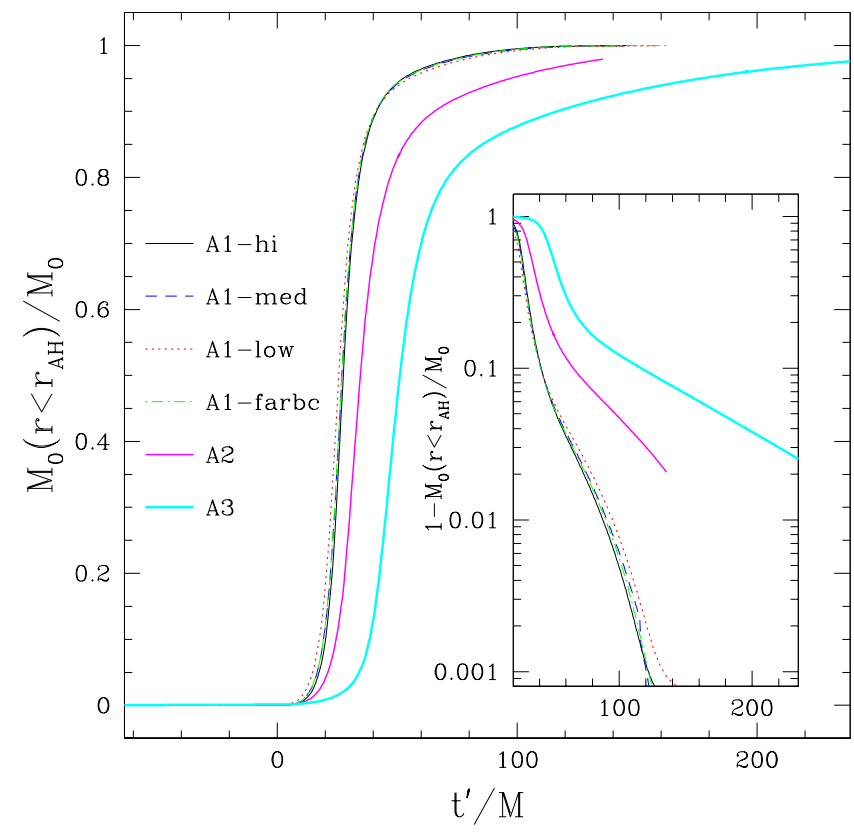

FIG. 3: Rest (baryon) mass fraction located inside the $\mathrm{BH}$ apparent horizon versus time for sequence $A$. Note that time is shifted $\left(t^{\prime} \equiv t-t_{\mathrm{FC}}\right)$ so that the first contact occurs at $t^{\prime}=0$. The inset shows the rest mass fraction outside the $\mathrm{BH}$ horizon at late times.

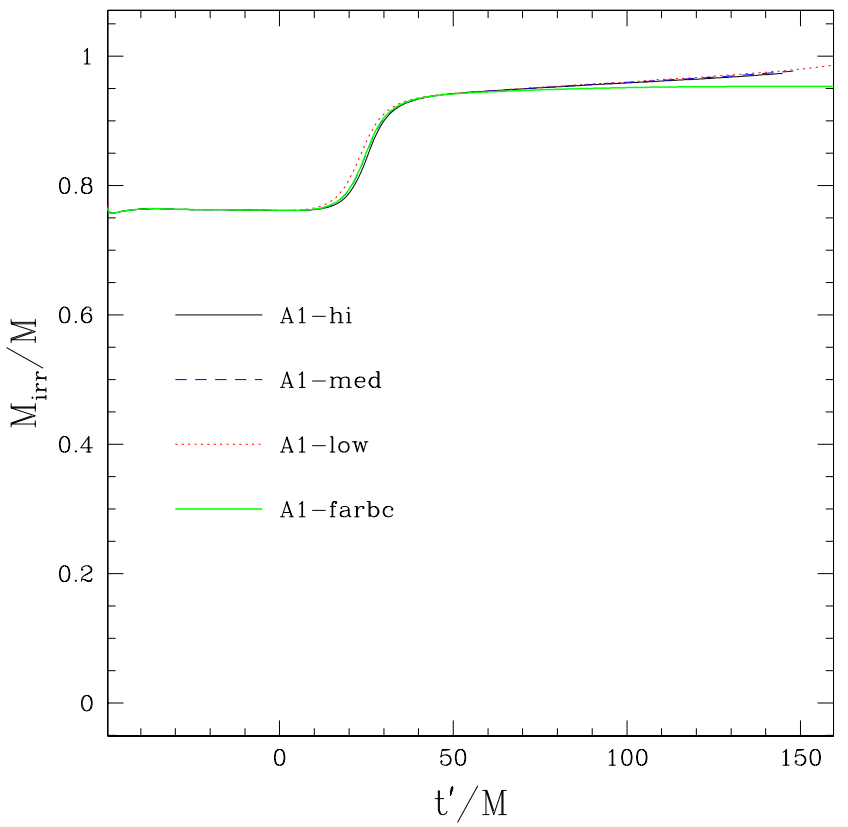

FIG. 4: Irreducible mass of the BH for sequence A1. Here $M$ is the total ADM mass.

has fallen into the $\mathrm{BH}$, and the accretion rate decreases considerably. Eventually the tail (Fig. 5) is swallowed by the $\mathrm{BH}$, but the accretion time scale is about twice as long as the $q=3$ case (Fig. 6).

At $t^{\prime} \approx 30 M$ in case $\mathrm{C}(q=1)$, a low-density region of matter develops ahead of the higher-density funnel region as $\chi^{\text {funnel }}$ becomes larger than $180^{\circ}$. As the majority of the NS material passes through the highdensity funnel, this low-density overshoot grows in size away from the horizon while wrapping quickly around the $\mathrm{BH}$, and finally smashing into the high-density funnel region (Fig. 5) before falling into the $\mathrm{BH}$. Once the low-density overshoot has been accreted $\left(t^{\prime} \approx 125 \mathrm{M}\right)$, only about $15 \%$ of the NS rest mass remains outside the $\mathrm{BH}$. The remaining NS matter is accreted at about the same rate as in the $q=3$ case.

We model our initial NS by a polytropic $\operatorname{EOS~} P=$ $\kappa \rho_{0}^{\Gamma}$ with $\Gamma=2$. During the evolution, shocks develop and the matter heats up, resulting in an increase in the parameter $K \equiv P /\left(\kappa \rho_{0}^{\Gamma}\right)$ from its initial value of unity. Fig. 7 plots contours of $K$ at different points in time for case C. Notice that $K$ is clearly larger than unity in the low-density overshoot region (upper-left panel of Fig. 7), and where the overshoot region smashes into the higherdensity funnel region (upper-right panel of Fig. 7). We also observe shock heating as the final bit of NS matter is accreted (lower-left panel of Fig. 7).

A variety of physical effects are not modeled in the post-shocked, semi-degenerate, nonequilibrium nuclear matter arising in these simulations. For example, transport due to photon and neutrino radiation is not modeled, so accurate measurements of temperature $T$ are not 

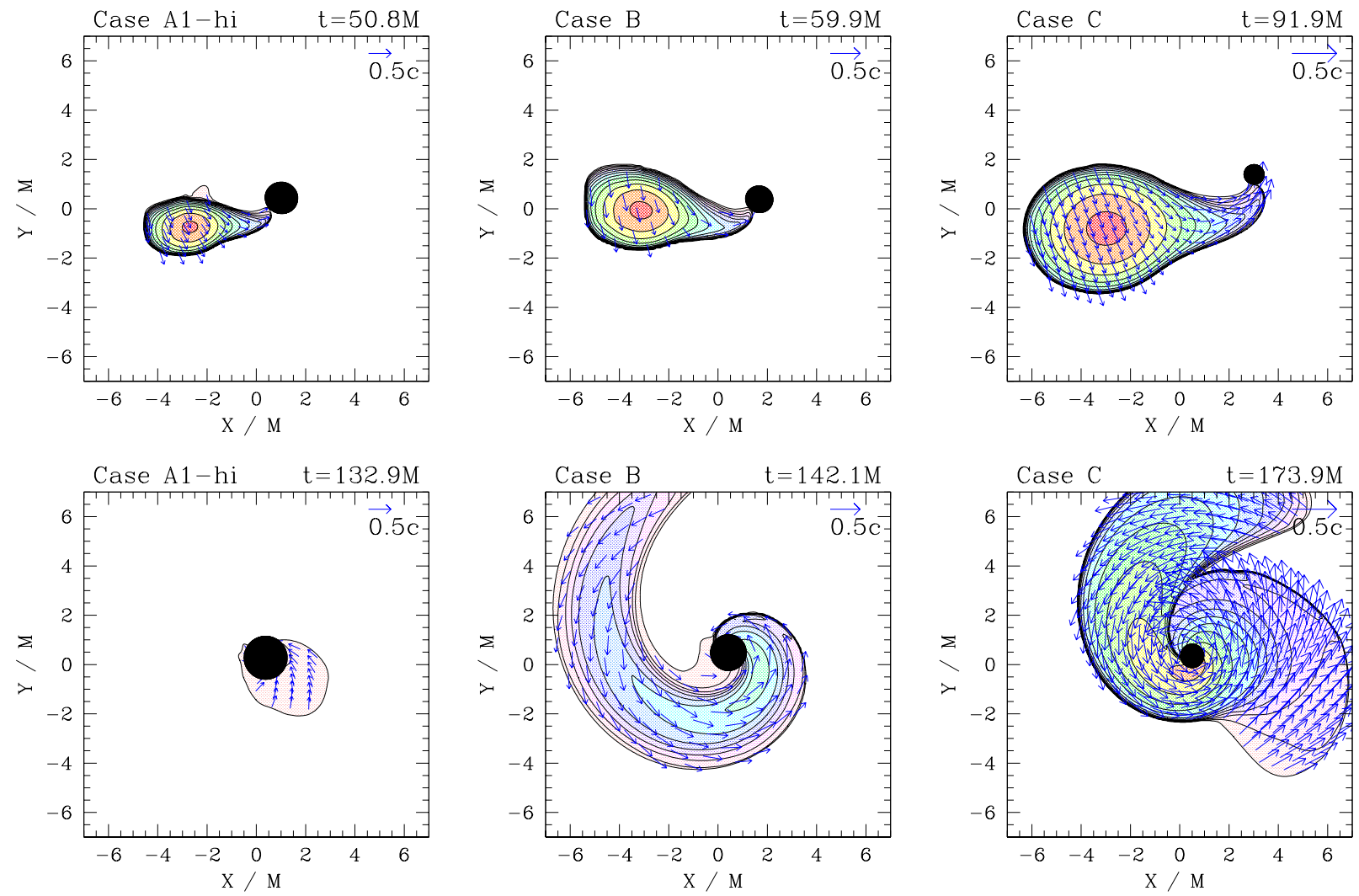

FIG. 5: Snapshots from runs A1-hi, B, and C, compared at first contact (upper panels) and at a moment in time $\Delta t \approx 75 M$ later (lower panels). Contours are defined as in Fig. 1.

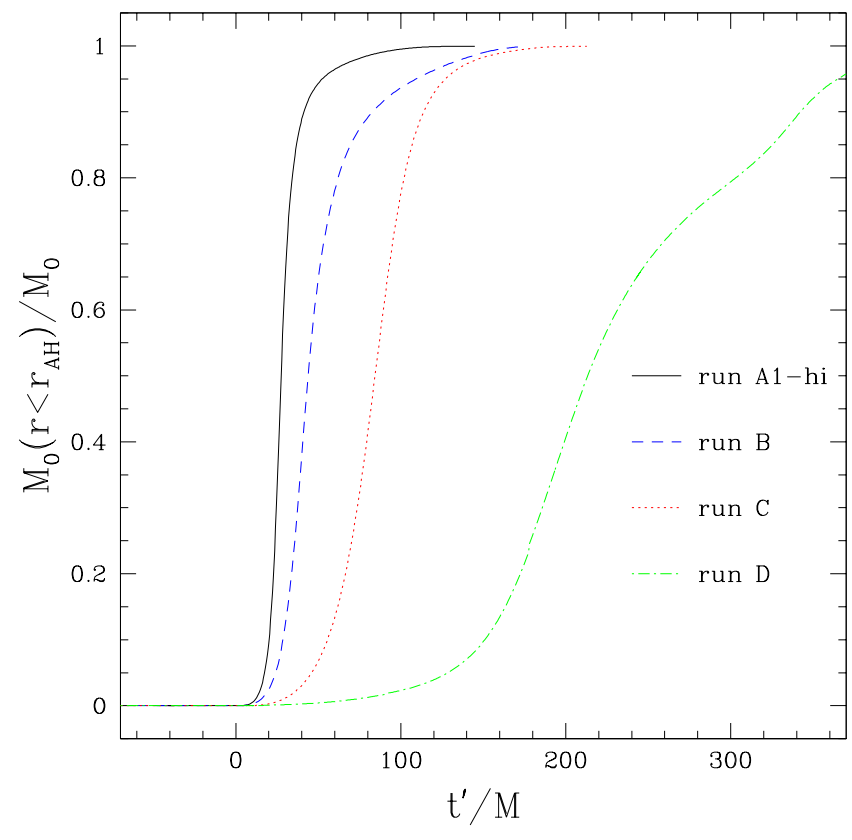

FIG. 6: Rest (baryon) mass located inside the $\mathrm{BH}$ apparent horizon versus $t^{\prime} / M$ for runs A1-hi, B, C, D. possible. Moreover, we are not employing a realistic hot nuclear EOS. However, we can very roughly estimate $T$ from the specific energy density $\epsilon$. For a polytropic equation of state, the "cold" contribution $\epsilon_{\text {cold }}$ is

$$
\epsilon_{\mathrm{cold}}=-\int P_{\text {cold }} d\left(1 / \rho_{0}\right)=\frac{\kappa}{\Gamma-1} \rho_{0}^{\Gamma-1},
$$

where $P_{\text {cold }} \equiv \kappa \rho_{0}^{\Gamma}$. We now define the thermal contribution to the specific energy density as $\epsilon_{\mathrm{th}}=\epsilon-\epsilon_{\mathrm{cold}}$ and compute the thermal contribution according to

$$
\begin{aligned}
\epsilon_{\mathrm{th}} & =\epsilon-\epsilon_{\mathrm{cold}}=\frac{1}{\Gamma-1} \frac{P}{\rho_{0}}-\frac{\kappa}{\Gamma-1} \rho_{0}^{\Gamma-1} \\
& =(K-1) \epsilon_{\mathrm{cold}},
\end{aligned}
$$

where we have used (25) to express $\epsilon$ in terms of $P$ and $\rho_{0}$.

To estimate $T$, we assume that we can model the temperature dependence of $\epsilon_{\mathrm{th}}$ as

$$
\epsilon_{\mathrm{th}}=\frac{3 k T}{2 m_{n}}+f \frac{a T^{4}}{\rho_{0}}
$$

(compare [92]), where $m_{n}$ is the mass of a nucleon, $k$ is the Boltzmann constant and $a$ is the radiation constant. The first term represents the approximate thermal energy of the nucleons, and the second term accounts for the thermal energy due to radiation. The 

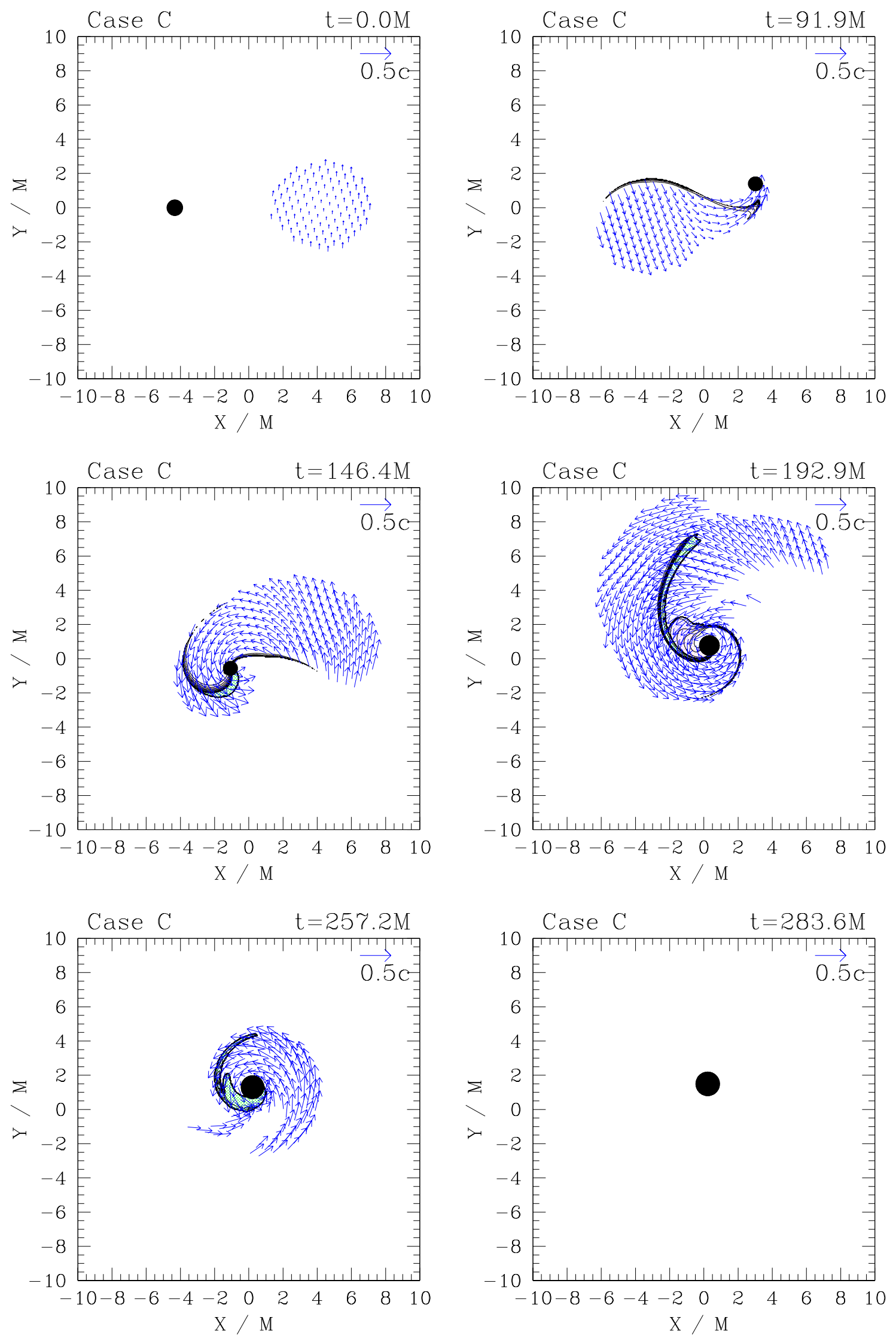

FIG. 7: Snapshots of equatorial contours of the polytropic constant $K$ at selected times for case C. Contours are spaced linearly, so that $\Delta K=0.89$ from $K=2$ to $K=10$. Values of $K>1$ result from shock heating; $K=1$ for adiabatic flow. 
factor $f$ reflects the number of species of ultrarelativistic particles that contribute to thermal radiation. When $T \ll 2 m_{e} / k \sim 10^{10} \mathrm{~K}$, where $m_{e}$ is the mass of electron, thermal radiation is dominated by photons and $f=1$. When $T \gg 2 m_{e} / k$, electrons and positrons become ultrarelativistic and also contribute to radiation, and $f=1+2 \times(7 / 8)=11 / 4$. At sufficiently high temperatures and densities $\left(T \gtrsim 10^{11} \mathrm{~K}, \rho_{0} \gtrsim 10^{12} \mathrm{~g} \mathrm{~cm}^{-3}\right)$, neutrinos are generated copiously and become trapped, so, taking into account three flavors of neutrinos and antineutrinos, $f=11 / 4+6 \times(7 / 8)=8$. For a heated region in the tidally disrupted NS, which possesses a typical density of $5 \times 10^{13} \mathrm{~g} \mathrm{~cm}^{-3}$, and $K \sim 7$, we obtain a temperature of $T \sim 2 \times 10^{11} \mathrm{~K}$. Although these temperatures are sufficient to produce copious neutrino emission, our low disk mass would limit the overall neutrino energy to $E_{\nu} \lesssim 10^{49}$ ergs (following the approximate scalings derived by [93] from numerical models of $\mathrm{BH}$ disks). This limits the total $\gamma$-ray annihilation energy to $\lesssim 10^{48}$ ergs assuming $10 \%$ efficiency, which may not be sufficient to power a SGRB. We note, however, that SGRB production may not require a long-lived massive disk, since the actual emission mechanism remains poorly understood. Instead, we merely require that sufficient thermal energy be produced to power the burst itself. So until a more detailed model of SGRB generation from BHNS mergers is developed, any assessment of these simulations regarding SGRBs is tentative at best.

In Fig. 8, we plot snapshots of case D, the lowcompaction, $q=2$ mass ratio case. At the time of first contact, the accretion funnel is much narrower than in the high-compaction cases of Fig. 5. Further, unlike any of the high-compaction cases, $\chi_{L}^{\text {funnel }}$ surpasses $180^{\circ}$ at $t^{\prime} \approx 165 M$ - about $60 M$ before the highest-density region of the NS has been accreted. After $\chi_{L}^{\text {funnel }}=180^{\circ}$, a case C-like overshoot develops (lower-left panel of Fig. 5), but instead of smashing into the higher-density funnel and quickly falling in to the $\mathrm{BH}$, the overshoot gently merges with the funnel to create a short-lived disk-like structure (lower-right panel of Fig. 5]) that later falls into the $\mathrm{BH}$.

The final disk masses we measure for each run are listed in Table III along with an estimate of the final (Kerr) $\mathrm{BH}$ spin. To calculate the latter quantity, we take the ratio of the polar to the equatorial circumference of the apparent horizon, $C_{r} \equiv C_{p} / C_{e}$ and use Eq. (5.3) of [94] to solve for the dimensionless spin $\tilde{a} \equiv a / M_{\mathcal{H}}$, where $M_{\mathcal{H}}=\left(M_{\mathrm{irr}} / \tilde{a}\right) \sqrt{2\left(1-\sqrt{1-\tilde{a}^{2}}\right)}$ is the Kerr BH ADM mass:

$$
C_{r}=\frac{1+\sqrt{1-\tilde{a}^{2}}}{\pi} E\left(-\frac{\tilde{a}^{2}}{\left(1+\sqrt{1-\tilde{a}^{2}}\right)^{2}}\right) .
$$

Here $E(x)$ is the complete elliptic integral of the second kind. In all cases, we find disk masses of less than $2.8 \%$ of the original NS mass. The final (Kerr) BH spin is roughly $a / M=0.5,0.64,0.8$, and 0.5 for cases $\mathrm{A}, \mathrm{B}, \mathrm{C}$, and $\mathrm{D}$, respectively. Here we use our finding that $M=M_{\mathcal{H}}$ to good approximation. The first of these agrees well with similar results of ST; this is the only case for which a meaningful comparison is possible, given the adopted mass ratios.

\section{Gravitational Wave emission}

The gravitational wavetrain from a compact binary system may be separated into three qualitatively different parts: the inspiral, merger, and ringdown. We describe each briefly before discussing our numerical results.

During the inspiral phase, which takes up most of the binary's lifetime, GW emission circularizes the orbit and gradually reduces the binary separation. At the large binary separations during the inspiral stage, finite-size effects associated with the NS are unimportant, and postNewtonian (PN) techniques are sufficient to describe the evolution. At present, the binary orbit dynamics is determined to 3.5PN order (e.g. 95]) and the corresponding GW emission is computed to $2.5 \mathrm{PN}$ order [96, 97] (but see also [98]). Even after finite-size corrections become relevant, quasi-equilibrium sequences allow for a determination of the binding energy as a function of orbital frequency, from which the GW energy spectrum $d E / d f$ may be calculated, following the techniques described in 99]. This method was used in [35] to determine the approximate energy spectrum from the sequences we use as initial data in this work.

Once the binary nears the ISCO, or the point where tidal disruption begins, the orbit decays rapidly and the GW emission changes character. In particular, deviations from point mass behavior typically result in a sharp decline in the energy spectrum. We note that these "break frequencies" marking the onset of instability systematically occur at lower frequencies for BHNS than for $\mathrm{BHBH}$ binaries (see discussion in [100]), especially in cases where tidal disruption occurs. This can be seen clearly in 36, noting that the tidal disruption branches do not exist for BHBH systems.

Finally, we expect a phase of quasinormal ringing of the $\mathrm{BH}$, since it is distorted by the merger. This emission typically results in a higher frequency peak in the energy spectrum, with an amplitude determined by the total distortion induced on the $\mathrm{BH}$ by the merger.

In Fig. 9, we plot the GW strains along the polar axis of the binary for case A1-hi, using both the $\psi_{4}$ formalism (solid curves; Eq. (49)) and the Z-M formualtion (dashed curves; Eq. (46)). In both cases the waveforms are extracted on a sphere of physical radius $r_{e x}=34.4 M$, and modes up to and including $l=4$ are used in the calculation. We add suitable integration constants when computing the waveforms from both Z-M (odd-parity modes) and $\psi_{4}$ formalisms to minimize offsets in the time-averaged $h_{+}$and $h_{\times}$. We see that there is some disagreement at early times as spurious gravitational radiation present in the initial data propagate outward. Once this "junk" radiation has left the numerical grid, the two independent methods yield results that are in very good 

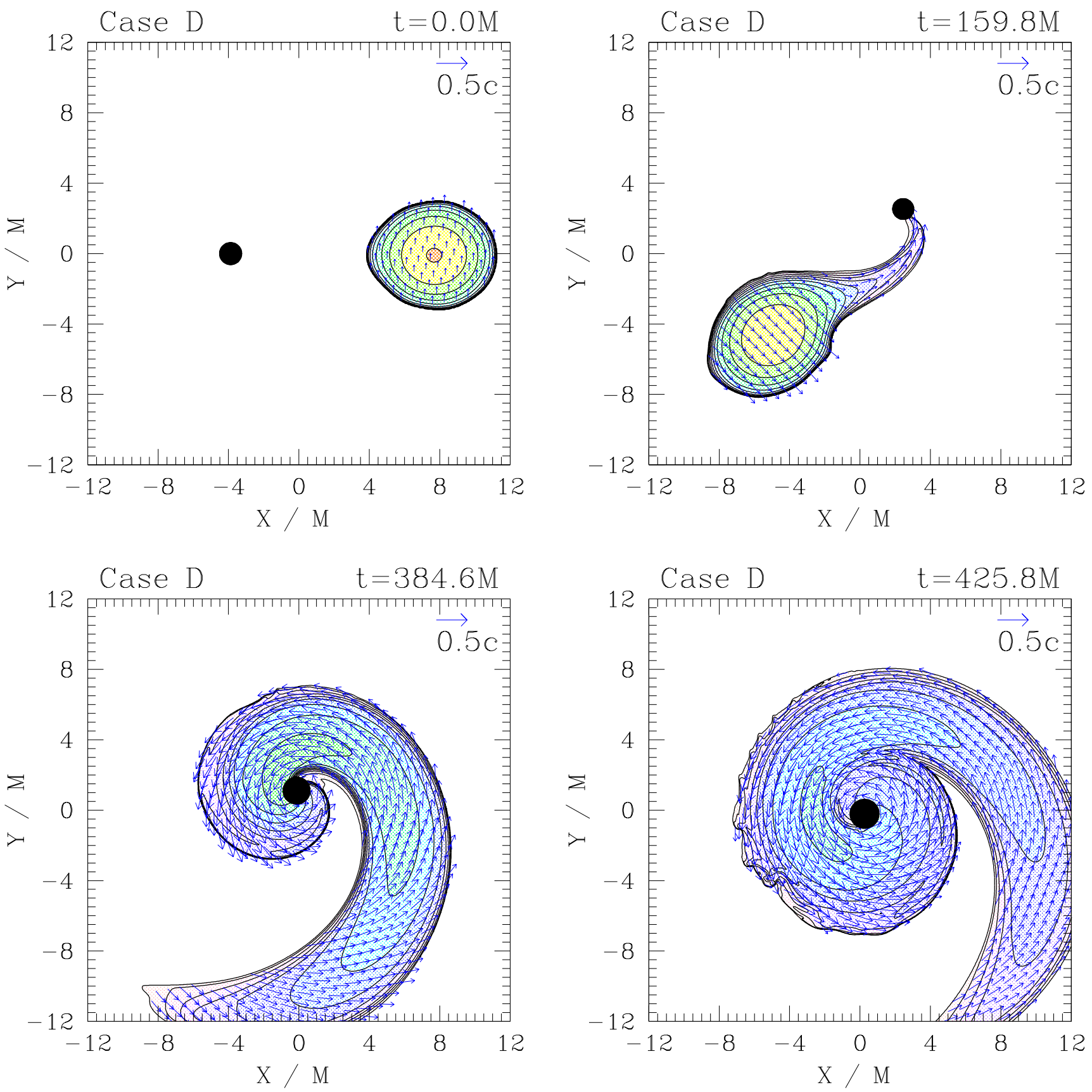

FIG. 8: Snapshots at selected times from case D, the low-compaction, $q=2$ mass ratio case. The contours represent the density in the orbital plane, plotted logarithmically with four contours per decade, with greyscaling added for clarity. Arrows represent the velocity field in the orbital plane. The minimum contour value in each frame is $\kappa \rho_{0}(\min )=10^{-4}$, or $\rho_{0}(\min )=$ $1.6 \times 10^{12}\left(1.4 M_{\odot} / M_{0}\right)^{2} \mathrm{~g} \mathrm{~cm}^{-3}$. The maximum initial NS density is $\kappa \rho_{0}=0.058$. We specify the black hole AH interior in each snapshot with a filled black circle. In cgs units, the total ADM mass for this case is $M=2 \times 10^{-5}\left(M_{0} / 1.4 M_{\odot}\right)$ $\mathrm{s}=6\left(M_{0} / 1.4 M_{\odot}\right) \mathrm{km}$.

agreement, even though they are calculated using different sets of metric components.

During the inspiral phase, the GW frequency and amplitude sweep upward until the point at which the NS begins to be disrupted by the $\mathrm{BH}$, at $t^{\prime}=0 M$. As accretion progresses, there is a gradual but steady downturn in the amplitude while the GW frequency continues to sweep upward. Finally, from $t^{\prime}=50 \mathrm{M}$ onward, after the vast majority of the NS matter has been accreted, we see a very weak ringdown signal, at amplitudes significantly less than those seen in either BHBH (30, 31, 101]) or NSNS ([15]) mergers. 
TABLE III: Final results from each of our simulations. We list the fractional rest (baryon) mass outside the horizon $f_{\text {out }} \equiv$ $M_{0}\left(r>r_{\mathrm{AH}}\right) / M_{0}$ and the dimensionless spin of the $\mathrm{BH} a / M$ [see Eq. (58)] at the end of our simulation. Also shown are the radiated energy, angular momentum, and linear velocity "kick" resulting from GW emission, the former two normalized to the binary's initial total ADM mass and the latter in km/s. For the GW quantities, entries without (with) parentheses are derived from the Z-M $\left(\psi_{4}\right)$ formalism. Note that in case D, the GW data are not accurate enough to obtain reliable estimate of $\Delta E_{\mathrm{GW}}$, $\Delta J_{\mathrm{GW}}$ and the kick velocity.

\begin{tabular}{|cccccc|}
\hline Case & $f_{\text {out }}$ & $a / M$ & $\Delta E_{\mathrm{GW}} / M$ & $\Delta J_{\mathrm{GW}} / M^{2}$ & Kick velocity $(\mathrm{km} / \mathrm{s})$ \\
\hline A1-lo & $<1 \%$ & $\approx 0.52$ & $0.60 \%(0.70 \%)$ & $5.2 \%(6.3 \%)$ & $21(21)$ \\
A1-med & $<1 \%$ & $\approx 0.52$ & $0.79 \%(0.75 \%)$ & $6.6 \%(6.2 \%)$ & $39(20)$ \\
A1-hi & $<1 \%$ & $\approx 0.52$ & $0.65 \%(0.77 \%)$ & $5.4 \%(6.4 \%)$ & $46(19)$ \\
A1-farbc & $<1 \%$ & $\approx 0.52$ & $0.74 \%(0.74 \%)$ & $6.0 \%(6.1 \%)$ & $15(16)$ \\
A2 & $<2 \%$ & $\approx 0.52$ & $0.72 \%(0.66 \%)$ & $7.3 \%(6.5 \%)$ & $49(24)$ \\
A3 & $<2.8 \%$ & $\approx 0.48$ & $0.67 \%(0.86 \%)$ & $7.9 \%(5.1 \%)$ & $18(30)$ \\
B & $<1 \%$ & $\approx 0.64$ & $0.59 \%(0.53 \%)$ & $6.2 \%(6.2 \%)$ & $67(48)$ \\
C & $<1 \%$ & $\approx 0.80$ & $0.39 \%(0.32 \%)$ & $5.4 \%(4.7 \%)$ & $22(25)$ \\
D & $<1 \%$ & $\approx 0.5$ & - & - & - \\
\hline
\end{tabular}

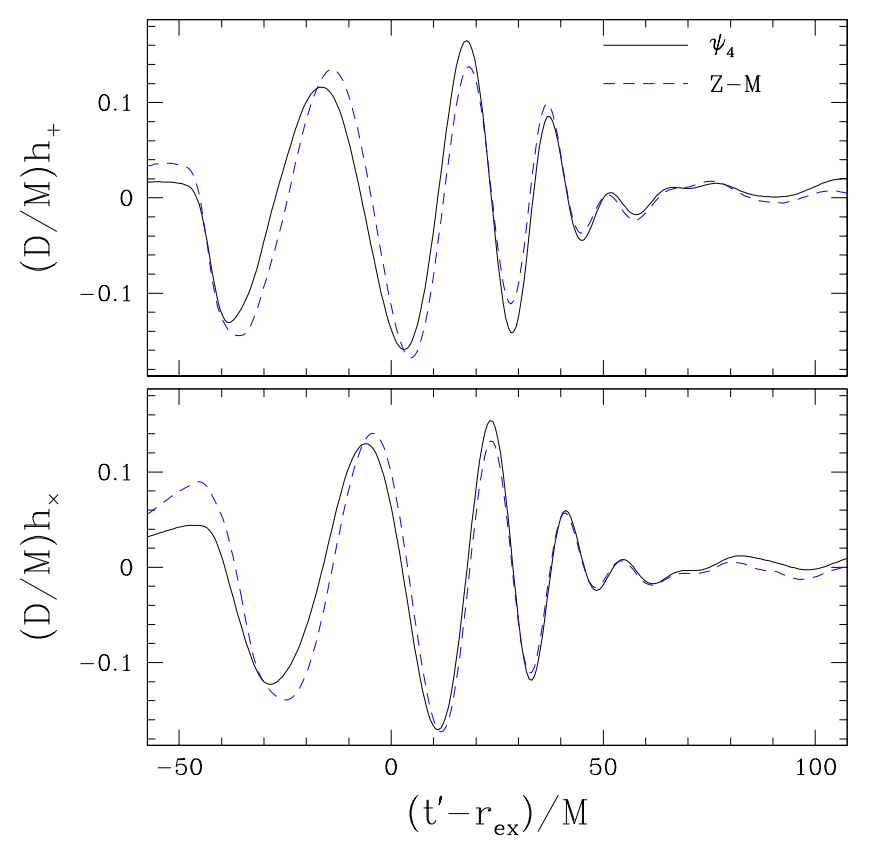

FIG. 9: Gravitational wave signal from case A1-hi, calculated using $\psi_{4}$ (solid line) and Z-M (dashed line). We show both polarizations as seen by an observer looking down the initial polar axis, $h_{+}$(top panel) and $h_{\times}$(bottom panel). The scale factor $D$ is the distance to the binary.

As discussed in Sec.IVA, the numerical resolution has little effect on the mass accretion rate in sequence $\mathrm{A}$. Correspondingly, we find that the tidal disruption signature in sequence A waveforms is largely resolutionindependent. For runs A1-hi, A1-med, and A1-low, we estimate the $\mathrm{GW}$ frequencies at $t^{\prime}-r_{e x}=0$ to be $M \Omega_{\mathrm{GW}} \equiv 2 \pi M f_{\mathrm{GW}}=0.188,0.180$ and 0.190 , respectively. These frequencies are slightly higher than twice the orbital frequency value at tidal disruption found in [35], $M \Omega_{\text {orb }} \approx 0.07$, as is expected since first contact occurs slightly after the onset of tidal disruption.

A similar pattern is observed in cases B and C (see Fig. (10). The ringdown amplitude grows relative to the overall signal strength as $q$ is reduced from 3 (sequence A) to 1 (case $\mathrm{C}$ ). However, in all cases the amplitude is significantly smaller than the comparable $\mathrm{BHBH}$ ringdown signal; we discuss this issue in more detail below.

The low compaction NS in case D implies a larger NS radius, so a larger initial binary separation was required than for the other cases. Thus, case D required many more grid light-crossing times until merger and ringdown. As a result, late-time normalized Hamiltonian constraint violation $\|\mathcal{H}\|$ increased to $\approx 8 \%$, leading to an inaccurate late-time waveform. We therefore truncate the waveform after $t^{\prime}-r_{e x}=200 M$. Based on our analysis of sequence $\mathrm{A}$ and the known high computational cost of case $\mathrm{D}$, accurate simulations of case $\mathrm{D}$ would require higher resolution and more distant outer boundaries than is practical, given our computational resources. Though we can still evolve the matter and trajectory of the BH reliably, we find that since the waveforms are manifested as small perturbations on the background spacetime, they are greatly affected by constraint violations.

We tabulate the GW energy loss $\Delta E_{\mathrm{GW}}$ and angular momentum loss $\Delta J_{\mathrm{GW}}$, as well as the measured kick velocity imparted to the $\mathrm{BH}$ in the rightmost columns of Table III Quantities without parentheses are derived from the Z-M formalism waveforms, and those in parentheses are derived from our $\psi_{4}$-based waveforms. We compute $\Delta E_{\mathrm{GW}}, \Delta J_{\mathrm{GW}}$, and the kick velocity at 5 radii in the range $\approx 31-37 M$ for all cases except A1-farbc (where the radii span $\approx 47-76 M$ ). The values shown in Table III are obtained by Richardson extrapolation of the data to $r \rightarrow \infty$. In general, we find good agreement between the two GW measurement methods, especially for the case with more distant outer boundaries, which satisfied the constraints best at late times. Based on the variations of results with different resolutions (for cases A1), different GW extraction radii and in the two GW ex- 

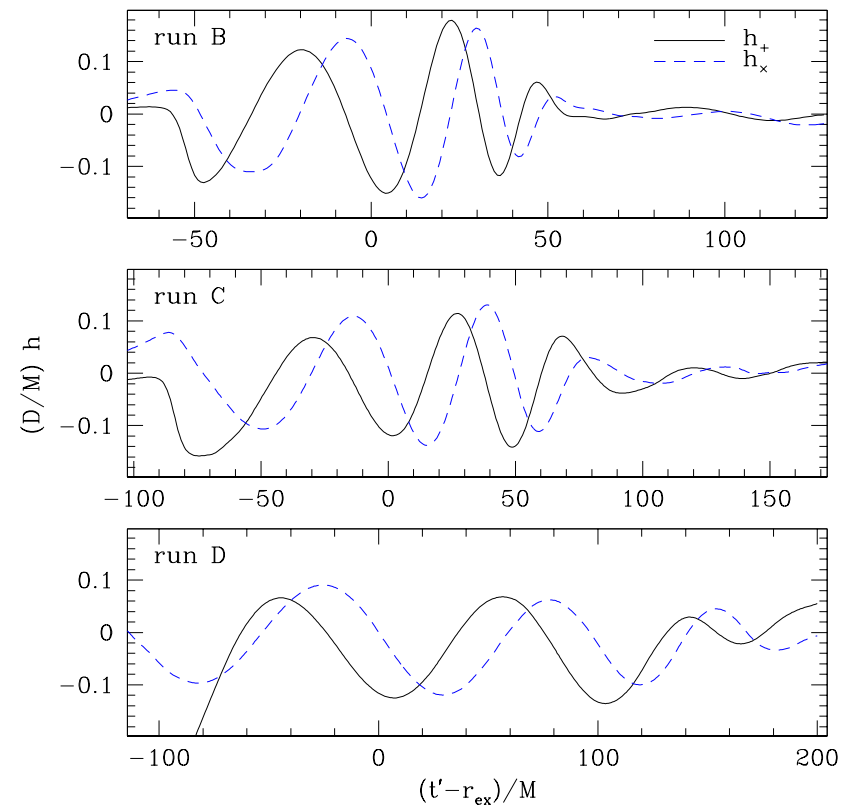

FIG. 10: Gravitational wave signal from cases B, C, and D, calculated using the Z-M formalism. We show both polarizations, $h_{+}$(solid) and $h_{\times}$(dashed) along the polar axis of the binary. The scale factor $D$ is the distance to the binary.

traction methods, we estimate that our tabulated $\Delta E_{\mathrm{GW}}$ and $\Delta J$ is accurate to about $20 \%$, whereas the error in kick velocity may be as much as $50 \%$. In case $\mathrm{D}$, the GW data are not accurate enough to provide reliable data for energy, angular momentum losses and kick velocity.

Compared to previous simulations of merging $\mathrm{BHBH}$ systems with the same mass ratio [102], we find that while the radiated energies, angular momenta and kick velocities are significantly lower in our runs because tidal disruption suppresses the GW signal, the final $\mathrm{BH}$ spins are comparable, within our uncertainties, to $\mathrm{BHBH}$ values.

In a previous work [38], we showed that our $\psi_{4}$ measurements converged to second-order with numerical resolution. In Fig. 11, we perform a similar demonstration, but with the Z-M formalism. In this figure, we plot the real component of the $\Psi_{\text {even }}^{22}$ mode for cases A1-hi, A1-med, and A1-low, which differ only in the numerical grid spacing. The three waveforms are plotted in the top panel and show good agreement. Notice that the waveform amplitudes are weakly dependent on the numerical resolution, but only at the level of a few percent for our higher resolution runs. In the bottom panel, we show differences between pairs of runs, rescaling the higher-resolution case by a numerical factor that assumes second-order convergence, $\left(166^{-2}-220^{-2}\right) /\left(220^{-2}-270^{-2}\right)=2.25$, finding agreement. Although our spatial differencing scheme for the fields is fourth-order accurate, our HRSC scheme in unshocked regions is only second-order accurate. This, along with the appearance of shocks (which are only

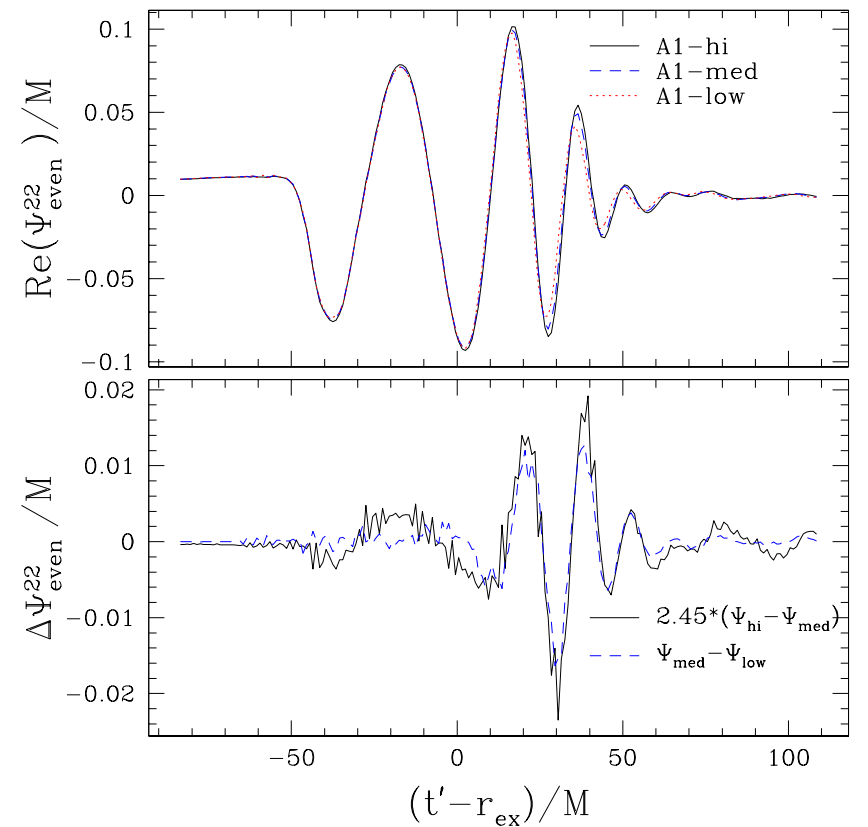

FIG. 11: Numerical convergence of gravitational wave signals for cases A1-hi, A1-med, and A1-lo. In the top panel, we show the $l=m=2$ component of the even-parity mode $\Psi_{\text {even }}^{22}$ for the three waveforms, noting that while they remain in phase with each other, we see overall amplitude differences on the order of several percent. In the bottom panel, pairwise differences between the waveforms are plotted, with the higher-resolution pair rescaled to demonstrate second-order convergence.

first-order convergent) limits the convergence order of our waveforms over time. As a result, while we can perform fourth-order time integrations with our code, we generally prefer second-order time integration since it is faster and does not result in a significant loss of accuracy.

Although the $l=2, m=2$ mode is the dominant component of the radiation, we measure all spin-weighted spherical harmonic components up to and including $l=$ 4. In Fig. 12, we show the mode decomposition of $\psi_{4}$ as a function of time for all non-negligible contributors. In the top panel of the figure, components satisfying $l=m$ are plotted, including the dominant $l=m=2$ mode. Notice that the modes satisfying $l=m+1$ in the middle panel possess an amplitude that is at most $15 \%$ of the total strain at any given moment. For completeness, we plot some of the other significant modes in the bottom panel, noting that while they are present in the initial passage of "junk" radiation, they play little or no role at later times.

To study the detectability and qualitative features of our computed gravitational wavetrains, we calculate the effective GW wave strain in frequency space using Eq. (54). We find that if an FFT is performed on these GW signals without modification, the initial burst of spurious junk radiation contributes significantly to the signal, and the finite initial amplitude introduces a strong 


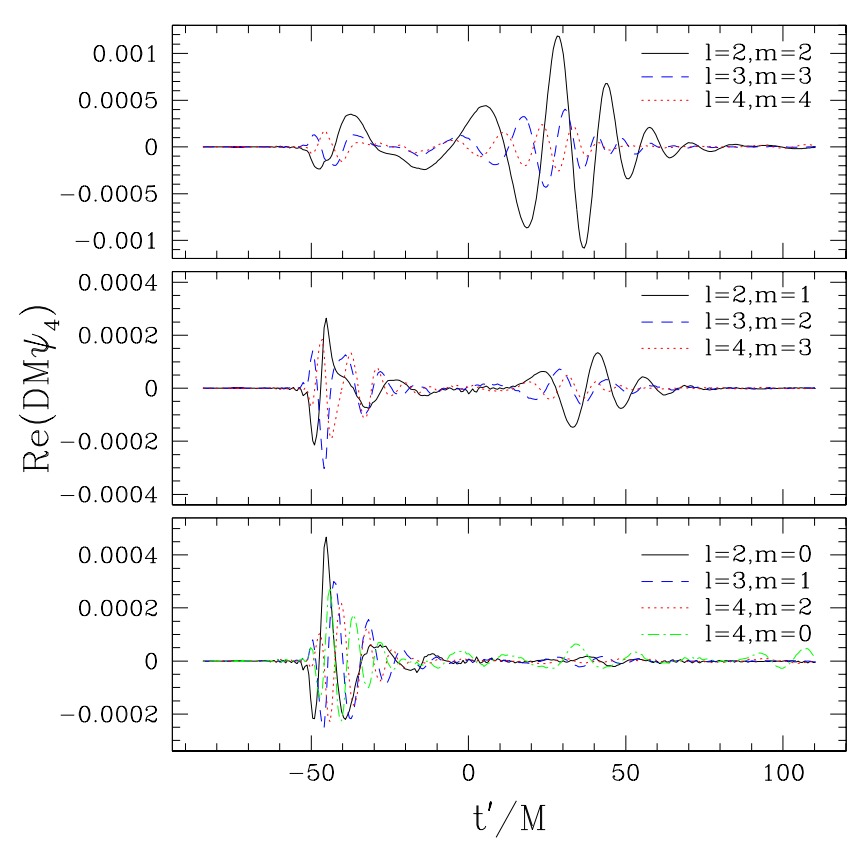

FIG. 12: Mode decompostition of the real component of $\psi_{4}$ for case A1-hi. In the top panel, we show the dominant $l=m$ modes. In the middle panel, we plot the modes that satisfy $l=m+1$, and in the bottom panel we show several other modes.

aliasing signal across the entire frequency domain of interest. To fix these problems, we perform "surgery" between our numerical GW signal, at a point in time where the initial junk has passed through the GW extraction surface, and a post-Newtonian signal with the same $q$. We generate the restricted PN waveform following the same techniques as in, e.g., [100]. Defining $v \equiv\left(\pi M f_{\mathrm{GW}}\right)^{1 / 3}$, we take as an initial condition the value of $v$ computed from the phase evolution of our numerical waveform and evolve backwards in time the following set of equations,

$$
\begin{aligned}
& \frac{d \varphi}{d t}=\frac{2 v^{3}}{M} \\
& \frac{d v}{d t}=-\frac{1}{M} \frac{F(v)}{d E(v) / d v}
\end{aligned}
$$

where the binding energy per unit mass $E(v)$ and radiation flux $F(v)$ are taken from the PN calculations of 95$]$. We modify the amplitude of the PN signal to minimize aliasing, but the relative correction is in all cases less than $2 \%$. Mismatches in the amplitude, frequency, and frequency sweep rate appear as oscillations in the energy spectrum near the surgery frequency. As a result, any peaks and troughs appearing at comparable frequencies in the energy spectrum should be viewed with skepticism unless demonstrated to be robust with respect to the surgery procedure. At both the beginning and end of the combined waveform, we add exponential damping terms to reduce aliasing, but this operation does not add power at frequencies of interest.

The result of this operation for run A3 is shown in Fig. 13, where units are set by assuming a NS rest mass of $1.4 M_{\odot}$. We plot the effective strain computed from both our combined waveform and our numerical signal alone. Notice that the signal follows the point-mass power law behavior up to frequencies of approximately $f_{\mathrm{GW}}=600-800 \mathrm{~Hz}$, at which point disruption of the $\mathrm{NS}$ and its subsequent accretion dominates the signal. At frequencies above $f_{\mathrm{GW}}=1 \mathrm{kHz}$, however, there is extremely little power in the waveform since the ringdown signal is so weak. These results are consistent with what we expect from the quasi-equilibrium configurations of [36], who find that $f_{\mathrm{GW}}=\Omega_{\mathrm{orb}} / \pi \approx 800 \mathrm{~Hz}$ for the configuration in question $\left(M f_{\mathrm{GW}}=0.022\right.$ in dimensionless units). They also agree roughly with results of [21], who find a similar pattern of steep decline above the tidal disruption value.

Each BHNS merger spectrum is compared to a BBH merger spectrum, taken from Eqs. (4.12)-(4.19) of [100], noting that $h_{\mathrm{eff}}(f) \propto f A(f)$ in their notation. The comparison is performed using a binary with the same masses as our BHNS case. Both of these curves lie above the advanced LIGO sensitivity band $h_{\mathrm{LIGO}}(f) \equiv \sqrt{f S_{h}(f)}$, which we have taken from [103]. This result assumes a distance to either source of $D=100 \mathrm{Mpc}$, the distance required to reach one merger per year assuming an overall rate of 10 mergers per megayear per Milky Wayequivalent galaxy (and a density of these of $0.1 \mathrm{gal} / \mathrm{Mpc}^{3}$ ) 104]. This distance is roughly that of the Coma cluster, and approximately five times the distance to the Virgo cluster. The difference in wave signal between BHBH and BHNS mergers is present in the advanced LIGO frequency band, but only marginally. It is clear that for more significant measurements of the difference between BHNS and BHBH inspirals and mergers, it would be advantageous to make use of narrow-band detection techniques with advanced detectors.

Fig. 14 contains plots of the GW spectrum for cases $\mathrm{B}, \mathrm{C}$, and $\mathrm{D}$. As the mass ratio and NS compaction is varied, we see the expected differences in the apparent "break frequency" marking tidal disruption. This frequency may be estimated by $M f_{\text {break }} \approx\left(M / d_{\text {tid }}\right)^{3 / 2} / \pi \approx$ $\mathcal{C}^{3 / 2}(1+q) \sqrt{(1+q) / q} / \pi$, where Eq. (11) has been used for $d_{\text {tid }}$. This formula is consistent with Eq. (25) of [36]. As the value of $q$ is lowered from 3.0 to 1.0, the break frequency rises. In addition, when we lower the compaction, we see a large decrease in the break frequency. These results, which agree well with the empirical scalings derived in [36], lend credence to the idea that if the individual masses of the binary components can be derived from the inspiral waveforms, the GW break frequency should provide a relatively sensitive measurement of the NS radius. When combined with observations of the lower-frequency spectrum, these inferences may provide additional constraints on the NS structure, including limits placed on the tidal Love number $k_{2}$ (following the techniques described in [105] for NSNS mergers). 


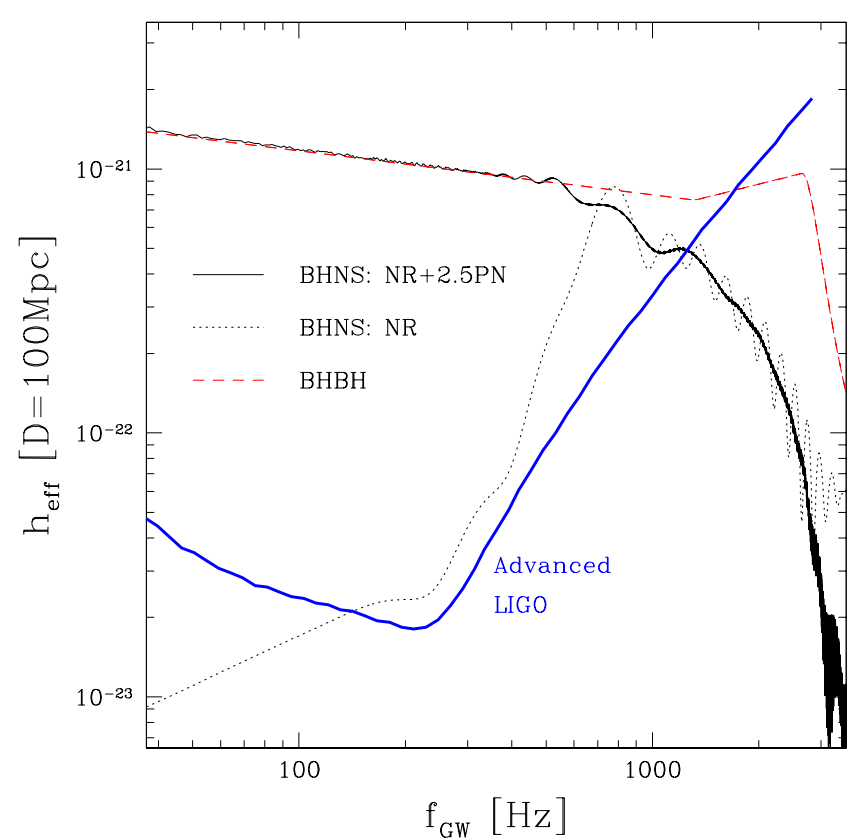

FIG. 13: Gravitational wave spectrum for the case A3 BHNS merger compared to a BHBH merger with the same masses. The solid curve shows the combined waveform found by attaching the restricted PN waveform to our numerical signal, while the dotted curve shows the contribution from the latter only, demonstrating the expected aliasing behavior resulting from FFTs of discontinuous functions. The dashed curve is the analytic fit derived by 100] from analysis of multi-orbit $\mathrm{BHBH}$ inspirals, which maintain significantly more power at higher frequencies. The heavy solid curve is the effective strain of the advanced LIGO detector, defined such that $h_{\text {LIGO }}(f) \equiv \sqrt{f S_{h}(f)}$. To set physical units, we assume a NS rest mass of $M_{0}=1.4 M_{\odot}$.

\section{DISCUSSION}

In this paper we present our first fully self-consistent, dynamical simulations of relativistic BHNS binaries. We use results and evolution techniques that we have previously developed in preparation for these simulations, including the initial data of [35], the "filling" of the black holes in these initial data [38] and the treatment of relativistic hydrodynamics in the context of the moving puncture method [37]. Here we focus on irrotational BHNS binaries with mass ratios between $q=1$ and 3 .

For the cases studied here, we find no more than $~$ $3 \%$ of the original NS matter remaining outside the $\mathrm{BH}$ at the end of the simulations. Such small disk masses lend support to the semi-analytic arguments presented in [53], which suggested that virtually the entire NS will be accreted promptly by the $\mathrm{BH}$.

The simulations of ST, on the other hand, suggest larger disk masses than ours. The reason for this discrepancy remains unclear, but we suspect it may be caused by different initial data - both choices of parameters and/or computational approach. We note that the mass

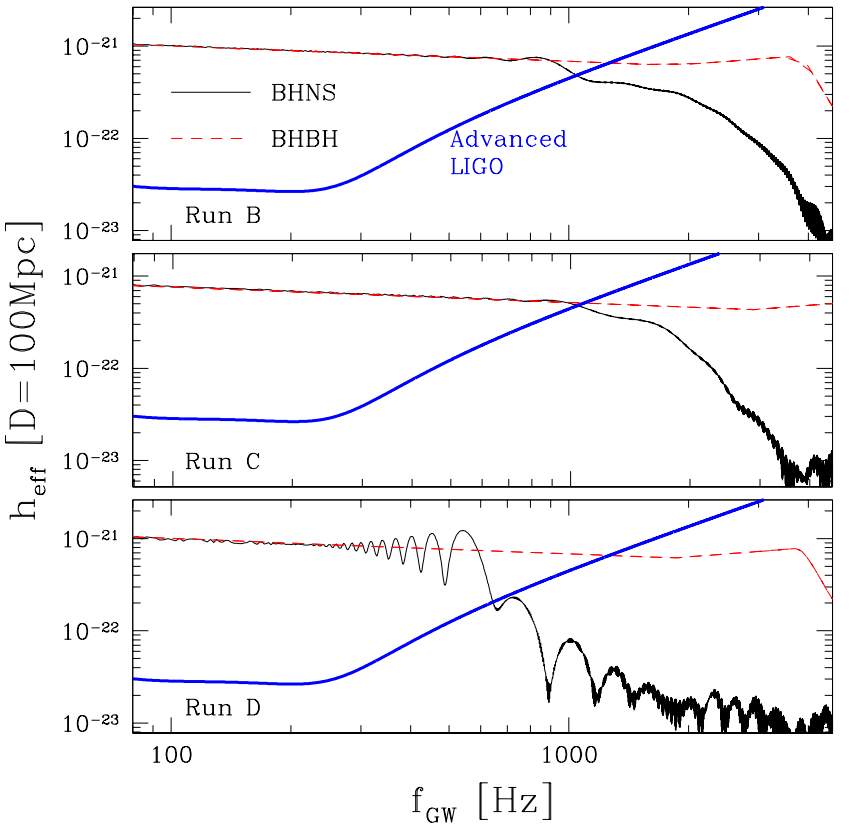

FIG. 14: Gravitational wave spectra for the cases B, C and D BHNS mergers compared to BHBH merger. Conventions are as in Fig. 13

accretion process depends on the initial binary separation (compare runs A1, A2 and A3 in Fig. 3), suggesting that the disk mass depends rather sensitively on the details of the initial data. However, we cannot rule out that this dependence is instead a numerical artifact, caused by numerical errors that are growing over time due to outer boundaries that are too close to the strong-field region and other effects. Since we model NSs as $\Gamma=2$ polytropes, and the disk mass is likely to depend sensitively on the NS EOS, firm conclusions about BHNS mergers as SGRB progenitors remain uncertain.

Our next series of BHNS simulations will involve spinning BHs. Most formation scenarios for BHNSs favor spinning BHs, especially systems in which the $\mathrm{BH}$ spin and orbital angular momenta axes are nearly aligned 106]. Since the ISCO for a prograde BH lies at a smaller radius than that of a non-spinning $\mathrm{BH}$, we expect that the tidal disruption of the NS around these BHs occurs farther from the ISCO. Thus, spinning BHs would likely lead to a more massive disk, but the magnitude of the effect and the scaling with respect to spin will need to be determined via numerical calculations (as suggested in 51], for the $q \simeq 10$ cases). Such calculations will enable us to probe in depth which areas in phase space are likely to serve as progenitors for SGRBs.

We find that the GW signal resulting from our BHNS coalescences is attenuated at frequencies roughly equal to double the orbital frequency at which tidal disruption begins, as one would expect, confirming the fits described in 36]. The deviation between BHNS and BHBH inspiral is visible in the advanced LIGO band for systems 
with mass ratios $q=3$ out to distances $\gtrsim 100 \mathrm{Mpc}$, within which volume some population synthesis calculations predict $\sim 1$ BHNS merger per year 104]. Should the chirp mass determination, combined with higher order PN waveform phase effects, allow for an independent determination of the component masses of the binary, observation of the BHNS merger break frequency should give a solid estimate of the NS radius. Such effects are independent of the discussion of disk formation, since the GW signal is strongly suppressed after the onset of tidal disruption.

We have performed a series of calculations for the $q=3$ mass ratio case (sequence A), in which we vary only grid parameters to determine the numerical resolution requirements for these BHNS mergers. Gross features of the hydrodynamics, such as the accretion rate onto the $\mathrm{BH}$, seem insensitive to the numerical resolution on the grid, at least for this case, where the NS accretes fairly promptly. The frequencies of the waveforms at critical moments are similarly insensitive to resolution. Waveform amplitudes, on the other hand, vary by a larger amount with respect to resolution, and are seen to be accurate only at times when the constraint violations remain small. At late times, when they are largest, constraint violations are dominated by finite boundary effects, which can be greatly reduced by enlarging the physical extent of the grid. Given the computational resource requirements for these simulations, it may be extremely costly to calculate waveforms accurate to a few percent using fisheye grids or similar fixed-mesh refinement techniques.
We expect that high accuracy calculations spanning $\sim 10$ orbits, as are currently performed in BHBH mergers, will require us to use adaptive mesh refinement (AMR) techniques. Our current technique includes a single high resolution grid that encompasses both the $\mathrm{BH}$, NS, and surrounding strong-field region. Outside of this region is a transition to a lower resolution grid domain that extends to the outer grid boundary. With AMR, we will be able to focus this high resolution entirely on the two regions immediately surrounding the $\mathrm{BH}$ and NS. With significantly fewer gridpoints in the strong-field region of the grid, we will be able to place more gridpoints in the low-resolution, weak-field region, thus extending our outer boundaries. In many ways, a relativistic hydrodynamics code with AMR will likely become a key tool for simulating BHNS spacetimes.

\section{Acknowledgments}

We thank V. Kalogera and S. Hughes for useful conversations, and the latter for providing a tabulated version of the advanced LIGO noise sensitivity curve. This paper was supported in part by NSF Grants PHY0205155, PHY03-45151, and PHY06-50377 as well as NASA Grants NNG04GK54G and NNX07AG96G to University of Illinois at Urbana-Champaign, and NSF Grant PHY04-56917 to Bowdoin College. JAF was also supported in part by NSF Grant AST04-01533. All simulations were performed on the NCSA abe cluster.
[1] B. Abbott and the LIGO Scientific Collaboration, ArXiv e-prints 704 (2007), 0704.3368.

[2] D. A. Brown, S. Babak, P. R. Brady, N. Christensen, T. Cokelaer, J. D. E. Creighton, S. Fairhurst, G. Gonzalez, E. Messaritaki, B. S. Sathyaprakash, et al., Class. Quant. Grav. 21, S1625 (2004).

[3] F. Acernese and the VIRGO Collaboration, Class. Quant. Grav. 23, 635 (2006).

[4] F. Beauville and the LIGO-VIRGO Working Group, ArXiv General Relativity and Quantum Cosmology eprints (2007), gr-qc/0701027.

[5] H. Lück and the GEO600 collaboration, Class. Quant. Grav. 23, S71 (2006).

[6] M. Ando and the TAMA collaboration, Class. Quant. Grav. 19, 1409 (2002).

[7] D. Tatsumi and the TAMA collaboration, Classical and Quantum Gravity 24, 399 (2007).

[8] G. Heinzel, C. Braxmaier, K. Danzmann, P. Gath, J. Hough, O. Jennrich, U. Johann, A. Rüdiger, M. Sallusti, and H. Schulte, Class. Quant. Grav. 23, 119 (2006).

[9] S. Kawamura and the DECIGO collaboration, Class. Quant. Grav. 23, 125 (2006).

[10] M. D. Duez, Y. T. Liu, S. L. Shapiro, M. Shibata, and B. C. Stephens, in Proceedings of the Eleventh Marcel Grossmann Meeting on General Relativity, ed. H. Klein- ert, R. T. Jantzen, and R. Ruffini (World Scientific, Singapore, 2007).

[11] B. C. Stephens, M. D. Duez, Y. T. Liu, S. L. Shapiro, and M. Shibata, in Proceedings of the Eleventh Marcel Grossmann Meeting on General Relativity, ed. H. Kleinert, R. T. Jantzen, and R. Ruffini (World Scientific, Singapore, 2007).

[12] M. Shibata, Y. T. Liu, S. L. Shapiro, and B. C. Stephens, Phys. Rev. D 74, 104026 (2006).

[13] M. Shibata, K. Taniguchi, and K. Uryū, Phys. Rev. D 68, 084020 (2003).

[14] M. Shibata, K. Taniguchi, and K. Uryū, Phys. Rev. D 71, 084021 (2005).

[15] M. Shibata and K. Taniguchi, Phys. Rev. D 73, 064027 (2006).

[16] M. Shibata, Y. Sekiguchi, and R. Takahashi, Prog. Theor. Phys. 118, 257 (2007).

[17] J. A. Faber, T. W. Baumgarte, S. L. Shapiro, K. Taniguchi, and F. A. Rasio, Phys. Rev. D 73, 024012 (2006).

[18] J. A. Faber, T. W. Baumgarte, S. L. Shapiro, and K. Taniguchi, Astrophys. J. Lett. 641, L93 (2006).

[19] M. Shibata and K. Uryū, Phys. Rev. D 74, 121503(R) (2006).

[20] M. Shibata and K. Uryū, Class. Quant. Grav. 24, 125 (2007). 
[21] M. Shibata and K. Taniguchi, ArXiv e-prints $\mathbf{7 1 1}$ (2007), 0711.1410.

[22] N. Gehrels, J. K. Cannizzo, and J. P. Norris, New Journal of Physics 9, 37 (2007).

[23] T. W. Baumgarte and S. L. Shapiro, Phys. Rep. 376, 41 (2003).

[24] M. Shibata, Phys. Rev. D 60, 104052 (1999), arXiv:grqc/9908027.

[25] M. Shibata and K. Uryū, Phys. Rev. D 61, 064001 (2000).

[26] P. Marronetti, M. D. Duez, S. L. Shapiro, and T. W. Baumgarte, Physical Review Letters 92, 141101 (2004), arXiv:gr-qc/0312036.

[27] M. Miller, P. Gressman, and W.-M. Suen, Phys. Rev. D 69, 064026 (2004), arXiv:gr-qc/0312030.

[28] M. Anderson, E. W. Hirschmann, L. Lehner, S. L. Liebling, P. M. Motl, D. Neilsen, C. Palenzuela, and J. E. Tohline, ArXiv e-prints 708 (2007), 0708.2720.

[29] F. Pretorius, Phys. Rev. Lett. 95, 121101 (2005).

[30] J. G. Baker, J. Centrella, D.-I. Choi, M. Koppitz, and J. van Meter, Phys. Rev. Lett. 96, 111102 (2006).

[31] M. Campanelli, C. O. Lousto, P. Marronetti, and Y. Zlochower, Phys. Rev. Lett. 96, 111101 (2006).

[32] T. W. Baumgarte, M. L. Skoge, and S. L. Shapiro, Phys. Rev. D 70, 064040 (2004).

[33] K. Taniguchi, T. W. Baumgarte, J. A. Faber, and S. L. Shapiro, Phys. Rev. D 72, 044008 (2005).

[34] K. Taniguchi, T. W. Baumgarte, J. A. Faber, and S. L. Shapiro, Phys. Rev. D 74, 041502 (2006).

[35] K. Taniguchi, T. W. Baumgarte, J. A. Faber, and S. L. Shapiro, Phys. Rev. D 75, 084005 (2007).

[36] K. Taniguchi, T. W. Baumgarte, J. A. Faber, and S. L. Shapiro, ArXiv e-prints (2007), 0710.5169.

[37] J. A. Faber, T. W. Baumgarte, Z. B. Etienne, S. L. Shapiro, and K. Taniguchi, Phys. Rev. D 76, 104021 (2007).

[38] Z. B. Etienne, J. A. Faber, Y. T. Liu, S. L. Shapiro, and T. W. Baumgarte, Phys. Rev. D 76, 101503(R) (2007).

[39] M. Miller, ArXiv General Relativity and Quantum Cosmology e-prints (2001), gr-qc/0106017.

[40] P. Grandclément, Phys. Rev. D 74, 124002 (2006).

[41] G. B. Cook and H. P. Pfeiffer, Phys. Rev. D 70, 104016 (2004).

[42] M. Caudill, G. B. Cook, J. D. Grigsby, and H. P. Pfeiffer, Phys. Rev. D 74, 064011 (2006).

[43] W. Tichy, B. Brügmann, and P. Laguna, Phys. Rev. D 68, 064008 (2003).

[44] R. Beig and N. Ó Murchadha, Class. Quantum Grav. 11, 419 (1994).

[45] R. Beig and N. Ó Murchadha, Class. Quantum Grav. 13, 739 (1996).

[46] S. Brandt and B. Brügmann, Phys. Rev. Lett. 78, 3606 (1997).

[47] W. H. Lee, Mon. Not. R. Astron. Soc. 328, 583 (2001).

[48] S. Rosswog, R. Speith, and G. A. Wynn, Mon. Not. R. Astron. Soc. 351, 1121 (2004).

[49] S. Rosswog, Astrophys. J. 634, 1202 (2005).

[50] S. Kobayashi, P. Laguna, E. S. Phinney, and P. Mészáros, Astrophys. J. 615, 855 (2004).

[51] E. Rantsiou, S. Kobayashi, P. Laguna, and F. Rasio, ArXiv Astrophysics e-prints (2007), astro-ph/0703599.

[52] F. Löffler, L. Rezzolla, and M. Ansorg, Phys. Rev. D 74, 104018 (2006).
[53] M. C. Miller, Astrophys. J. Lett. 626, L41 (2005).

[54] J. A. Isenberg, ArXiv General Relativity and Quantum Cosmology e-prints (2007), gr-qc/0702113.

[55] J. R. Wilson, G. J. Mathews, and P. Marronetti, Phys. Rev. D 54, 1317 (1996).

[56] B. Brügmann, Int. J. Mod. Phys. D 8, 85 (1999).

[57] B. Brügmann, W. Tichy, and N. Jansen, Phys. Rev. Lett. 92, 211101 (2004).

[58] M. Shibata and T. Nakamura, Phys. Rev. D 52, 5428 (1995).

[59] T. W. Baumgarte and S. L. Shapiro, Phys. Rev. D 59, 024007 (1998).

[60] M. Alcubierre, B. Brügmann, P. Diener, M. Koppitz, D. Pollney, E. Seidel, and R. Takahashi, Phys. Rev. D 67, 084023 (2003).

[61] C. Bona, J. Massó, E. Seidel, and J. Stela, Phys. Rev. Lett. 75, 600 (1995).

[62] M. Hannam, S. Husa, D. Pollney, B. Brügmann, and N. Ó Murchadha, ArXiv e-prints (2006), gr-qc/0606099.

[63] M. Hannam, S. Husa, B. Brügmann, J. A. González, U. Sperhake, and N. Ó Murchadha, J. Phys. Conf. Series 66, 012047 (2007).

[64] J. D. Brown, ArXiv e-prints 705 (2007), 0705.1359.

[65] T. W. Baumgarte and S. G. Naculich, Phys. Rev. D 75, 067502 (2007).

[66] M. D. Duez, Y. T. Liu, S. L. Shapiro, and B. C. Stephens, Phys. Rev. D 72, 024028 (2005).

[67] T. W. Baumgarte, Phys. Rev. D 62, 024018 (2000).

[68] D. Brown, O. Sarbach, E. Schnetter, M. Tiglio, P. Diener, I. Hawke, and D. Pollney, Phys. Rev. D 76, 081503 (2007).

[69] K. Belczynski, V. Kalogera, and T. Bulik, Astrophys. J. 572, 407 (2002).

[70] K. Belczynski, R. E. Taam, E. Rantsiou, and M. van der Sluys, ArXiv Astrophysics e-prints (2007), astro$\mathrm{ph} / 0703131$.

[71] V. Kalogera, K. Belczynski, C. Kim, R. O'Shaughnessy, and B. Willems, Phys. Rept. 442, 75 (2007).

[72] M. D. Duez, P. Marronetti, S. L. Shapiro, and T. W. Baumgarte, Phys. Rev. D 67, 024004 (2003).

[73] J. R. van Meter, J. G. Baker, M. Koppitz, and D.-I. Choi, Phys. Rev. D 73, 124011 (2006).

[74] B. van Leer, Journal of Computational Physics 23, 276 (1977).

[75] A. Harten, P. Lax, and B. van Leer, SIAM Rev. 25, 35 (1983).

[76] http://www. cactuscode.org/.

[77] M. Campanelli, C. O. Lousto, and Y. Zlochower, Phys. Rev. D 73, 061501 (2006).

[78] Y. Zlochower, J. G. Baker, M. Campanelli, and C. O. Lousto, Phys. Rev. D 72, 024021 (2005).

[79] M. D. Duez, S. L. Shapiro, and H.-J. Yo, Phys. Rev. D 69, 104016 (2004).

[80] J. Thornburg, Class. Quant. Grav. 21, 743 (2004).

[81] F. J. Zerilli, Phys. Rev. D 2, 2141 (1970).

[82] V. Moncrief, Annals of Physics 88, 323 (1974).

[83] M. Ruiz, R. Takahashi, M. Alcubierre, and D. Nunez, ArXiv e-prints 707 (2007), 0707.4654.

[84] M. Shibata and Y.-I. Sekiguchi, Phys. Rev. D 71, 024014 (2005).

[85] É. É. Flanagan and S. A. Hughes, Phys. Rev. D 57, 4535 (1998).

[86] M. Shibata, Phys. Rev. Lett. 94, 201101 (2005). 
[87] G. B. Cook, S. L. Shapiro, and S. A. Teukolsky, Astrophys. J. 398, 203 (1992).

[88] M. Miller, Phys. Rev. D 69, 124013 (2004).

[89] E. Berti, S. Iyer, and C. M. Will, Phys. Rev. D 74, 061503 (2006), arXiv:gr-qc/0607047.

[90] H. P. Pfeiffer, D. A. Brown, L. A. Kidder, L. Lindblom, G. Lovelace, and M. A. Scheel, Class. Quant. Grav. 24, S59 (2007).

[91] S. Husa, M. Hannam, J. A. Gonzalez, U. Sperhake, and B. Bruegmann, ArXiv e-prints 706 (2007), 0706.0904.

[92] R. Popham, S. E. Woosley, and C. Fryer, Astrophys. J. 518, 356 (1999).

[93] S. Setiawan, M. Ruffert, and H.-T. Janka, Astron. \& Astrophys. 458, 553 (2006).

[94] M. Alcubierre, B. Brügmann, P. Diener, F. S. Guzmán, I. Hawke, S. Hawley, F. Herrmann, M. Koppitz, D. Pollney, E. Seidel, et al., Phys. Rev. D 72, 044004 (2005).

[95] L. Blanchet, G. Faye, B. R. Iyer, and B. Joguet, Phys. Rev. D 65, 061501 (2002), 71, 129902(E) (2005).

[96] K. G. Arun, L. Blanchet, B. R. Iyer, and M. S. S. Qusailah, Classical and Quantum Gravity 21, 3771 (2004), arXiv:gr-qc/0404085.
[97] L. E. Kidder, L. Blanchet, and B. R. Iyer, Classical and Quantum Gravity 24, 5307 (2007), arXiv:0706.0726.

[98] L. E. Kidder, ArXiv e-prints 710 (2007), 0710.0614.

[99] J. A. Faber, P. Grandclément, F. A. Rasio, and K. Taniguchi, Phys. Rev. Lett. 89, 231102 (2002).

[100] P. Ajith, S. Babak, Y. Chen, M. Hewitson, B. Krishnan, A. M. Sintes, J. T. Whelan, B. Bruegmann, P. Diener, N. Dorband, et al., ArXiv e-prints 710 (2007), 0710.2335.

[101] B. Bruegmann, J. Gonzalez, M. Hannam, S. Husa, and U. Sperhake, ArXiv e-prints 707 (2007), 0707.0135.

[102] J. A. González, U. Sperhake, B. Brügmann, M. Hannam, and S. Husa, Phys. Rev. Lett. 98, 091101 (2007).

[103] E. Gustafson, D. Shoemaker, K. Strain, and R. Weiss, LSC White Paper on Detector Research and Development (1999), LIGO project document T990080-00-D.

[104] K. Belczynski, T. Bulik, and B. Rudak, Astrophys. J. 571, 394 (2002).

[105] E. E. Flanagan and T. Hinderer, ArXiv e-prints 709 (2007), 0709.1915.

[106] J. D. Schnittman, Phys. Rev. D 70, 124020 (2004). 